\title{
An isogeometric collocation method for frictionless contact of Cosserat rods
}

\author{
Oliver Weeger ${ }^{\mathrm{a}, *}$, Bharath Narayanan ${ }^{\mathrm{a}}$, Laura De Lorenzis ${ }^{\mathrm{b}}$, Josef Kiendl ${ }^{\mathrm{c}}$, Martin L. Dunn $^{\mathrm{a}}$ \\ ${ }^{a}$ Singapore University of Technology and Design, SUTD Digital Manufacturing and Design Centre, \\ 8 Somapah Road, Singapore 487372, Singapore \\ ${ }^{b}$ Technische Universität Braunschweig, Institut für Angewandte Mechanik, \\ Bienroder Weg 87, 38106 Braunschweig, Germany \\ ${ }^{c}$ Norwegian University of Science and Technology, Department of Marine Technology, \\ NTNU, 7491 Trondheim, Norway
}

\begin{abstract}
A frictionless contact formulation for spatial rods is developed within the framework of isogeometric collocation. The structural mechanics is described by the Cosserat theory of geometrically nonlinear spatial rods. The numerical discretization is based on an isogeometric collocation method, where the geometry and solution fields are represented as NURBS curves and the strong forms of the equilibrium equations are collocated at Greville points. In this framework, a frictionless rod-to-rod contact formulation is proposed. Contact points are detected by a coarse-level and a refined search for close centerline points and reaction forces are computed by the actual penetration of rod surface points, so that the enforcement of the contact constraints is performed with the penalty method. An important aspect is the application of contact penalty forces as point loads within the collocation scheme, and methods for this purpose are proposed and evaluated. The overall contact algorithm is validated by and applied to several numerical examples.
\end{abstract}

Keywords: Isogeometric analysis, Collocation method, Contact formulation, Nonlinear rods

\section{Introduction}

Mechanical simulation of 3D beams, rods and structures undergoing large deformations has a wide range of applications in modern product development and design processes. For complex applications, such as the simulation of yarns [1], woven and knitted textiles [2], fibrous materials [3], hair, cables [4, pipings or additively manufactured structures [5, the capability to simulate rod-to-rod contact is essential. In this paper, we present an isogeometric collocation method for contact simulation of geometrically nonlinear spatial rods, which exploits the advantages of isogeometric collocation methods, i.e. numerical accuracy and efficiency, as well as integration into computer-aided design, for these applications.

Isogeometric analysis (IGA), first proposed in [6], has gained significant popularity in practically all fields of computational mechanics, in particular in structural mechanics where many new formulations for beam, plate, and shell analysis have been proposed. IGA for beams was first explored in 7 with the study of Bernoulli-Euler beam vibrations. Later, IGA of nonlinear Bernoulli-Euler beam vibrations was presented in [8]. The first isogeometric implementation for fully 3D Kirchhoff rods was reported in [9], and 10 developed a geometrically nonlinear formulation. All of the above works were based on the socalled thin beam theories, where shear deformation is neglected. Locking-free isogeometric formulations for curved thick beams were presented in [11, whereas in [12] a formulation for shear-deformable beams with only one unknown variable was proposed. Recently, object-oriented C++ libraries for the implementation of isogeometric methods have been introduced with igatools [13] and G+SMo [14], the latter being also the foundation of the implementation in this paper.

\footnotetext{
* Corresponding author

Email address: oliver_weeger@sutd.edu.sg (Oliver Weeger)
} 
An important feature of IGA for all the aforementioned formulations is the high inter-element continuity provided by the isogeometric basis functions. This property is also the basis for the recent development of isogeometric collocation methods (IGA-C), an alternative to Galerkin formulations, where the discretized governing differential equations of a given problem, as well as the Neumann boundary conditions, are enforced in strong form 15, 16 at appropriate collocation points, which are typically chosen as the Greville or Demko abscissae of the knot vectors. These are equal in number to the control points and some are always located on the boundary of the domain. The optimal choice of the collocation points is an aspect on which very recent advances have been reported [17, 18.

A distinct feature of the collocation approach is that it requires only one point evaluation per unknown, which makes it significantly faster than Galerkin methods, especially for problems where the computational cost is directly related to quadrature, e.g., in explicit dynamics [19. A comprehensive study and comparison of IGA, IGA-C, and the standard (Galerkin) finite element method (FEM) in terms of accuracy and efficiency can be found in 20. Isogeometric collocation has been successfully applied to different problems of solid and structural mechanics. Of particular relevance for the present work are the investigations on straight Timoshenko beams 21] and spatial Timoshenko rods [22, Reissner-Mindlin plates [23], Bernoulli beams and Kirchhoff plates 24, and frictionless contact 25]. In 26, isogeometric collocation was extended to nonlinear problems, studying large deformation elasticity and frictional contact. Formulations for geometrically nonlinear Cosserat rods and rod structures were presented in 27] and [28].

An overview of contact formulations within IGA was provided in 29] and showed that the main aspects inherent to the contact formulations themselves, such as patch test performance, stability and computational efficiency, do not change when passing from the FEM to the IGA setting. However, all disadvantages emanating from the non-smooth discretization are naturally avoided in IGA, so that the robustness and the overall performance appear far superior to those of the same discretizations in conventional FEM. Contact formulations suitable for the IGA-C framework in elasticity were derived in [25] and [26] for the frictionless and frictional cases, respectively. In this setting, the most natural approach to enforce the contact constraints is to treat them as deformation-dependent Neumann boundary conditions on the portion of the boundary in active contact, which may be identified with classical active set strategies.

While contact to plates and shells can be addressed with minor modifications with respect to the standard contact between solids, beam contact requires ad hoc contact procedures. The problem was initially treated in [30, 31] for beams with circular cross-sections. There, contact between two beams was assumed to be pointwise and defined by a minimum distance criterion. This approach was extended in [32, 33] to consider contact between beams with rectangular cross-sections. On the same basis of pointwise contact, 34 presented a geometrically exact theory for contact interactions of $1 \mathrm{D}$ manifolds in $3 \mathrm{D}$, using various types of approximations, including isogeometric beam elements. In a knot tying example, iterative convergence could only be achieved with the latter. A recent formulation for pointwise beam-to-beam contact with an unbiased treatment of the contacting surfaces was proposed in 35].

In situations where the contact zone between two beams is expected to be continuous, for instance because two beams are almost parallel or bent on each other, the notion of minimum distance becomes less relevant to characterize and locate contact. A solution is to follow a master/slave strategy, as proposed in 36] in the context of self-contact within a single beam. In 37. two contact points were added at a given distance on both sides of the point of minimal distance. In [1] contact was considered as a phenomenon involving two beams symmetrically with respect to an intermediate entity. This approach was applied to contact of entangled fibrous materials [2, 3, to predict the mechanical behavior of superconducting cables 4, as well as for the simulation of the tightening of knots made of monofilament and multifilament yarns 1. A unified method for point and line load based beam-to-beam contact was recently proposed in [38, 39. Self-contact of Cosserat rods was addressed in [36, 40, whereas self-contact in beams experiencing loop formation was studied in [41.

In this paper, we propose a formulation for frictionless contact between Cosserat rods within the framework of isogeometric collocation. The geometry and solution fields are represented by NURBS curves and the equilibrium equations of linear and angular momentum are discretized and collocated in strong form along with the corresponding boundary conditions. Within the frictionless contact formulation, the geometric contact detection is conducted with a three-stage approach and is followed by the computation of the 


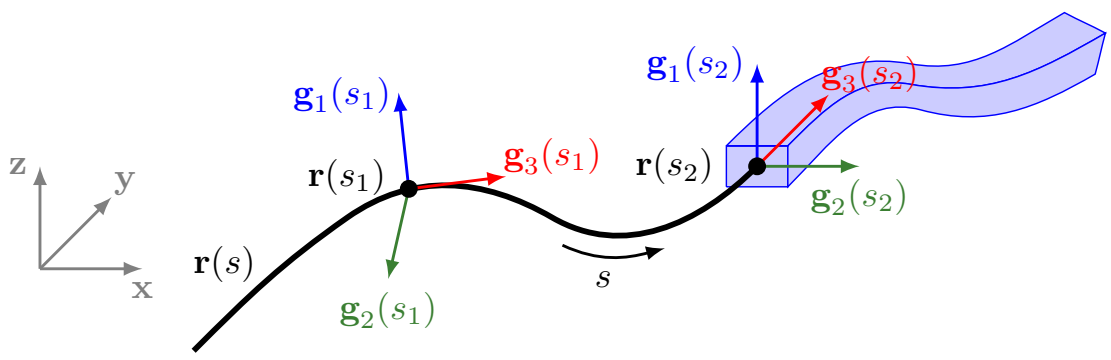

Figure 1: Configuration of the Cosserat rod as a framed curve with centerline $\mathbf{r}(s)$ and orthonormal frames $\mathbf{R}(s)=$ $\left(\mathbf{g}_{1}(s), \mathbf{g}_{2}(s), \mathbf{g}_{3}(s)\right)$ defining the cross-section orientations (see [27])

contact forces and the enforcement of the contact constraints using the penalty method. Special attention is devoted to the issue of point loads within the IGA-C scheme, which stems from the collocated nature of the contact forces but is a more general issue in collocation schemes. The contact algorithm is demonstrated with several numerical applications. The structure of the paper is as follows: Sections 2 and 3 review the Cosserat rod model and the isogeometric collocation method, respectively; Section 4 illustrates the proposed contact formulation, including its geometric and mechanical components, addresses the issue of point loading, and overviewes the algorithmic and implementation aspects; in Section 5, several numerical examples of increasing complexity are presented. The main conclusions are drawn in Section 6

\section{Cosserat rod model}

In this section we briefly introduce the Cosserat rod model, which we use for the mechanical description of slender, elastic, 3D rods 42 44. The Cosserat rod theory can be seen as a nonlinear extension of the spatial Timoshenko beam model, and is thus also based on the assumption that the cross-sections remain undeformed, but not necessarily normal to the tangent of the centerline curve, which accounts also for shear deformation.

In the Cosserat model, a rod is represented as a framed curve (see Fig. 11) and thus its configuration is completely described by its centerline curve, i.e. the line of its mass centroids,

$$
\mathbf{r}:[0, L] \rightarrow \mathbb{R}^{3},
$$

and a frame, or local orthonormal basis field:

$$
\mathbf{R}:[0, L] \rightarrow S O(3)
$$

The centerline curve is arc-length parameterized in the initial configuration, which means that $\left\|\mathbf{r}^{\prime}(s)\right\|=$ $\left\|\frac{d \mathbf{r}}{d s}\right\|=1 \forall s \in[0, L]$ and thus $L=\int_{0}^{L}\left\|\mathbf{r}^{\prime}(s)\right\| d s$ is the initial length of the curve. The local frames describe the evolution of the orientation of the cross-section and can be associated with 3D rotation matrices $\mathbf{R}(s)=\left(\mathbf{g}_{1}(s), \mathbf{g}_{2}(s), \mathbf{g}_{3}(s)\right) \in \mathbb{R}^{3 \times 3}: \mathbf{R}^{\top} \mathbf{R}=\mathbf{I}$, $\operatorname{det} \mathbf{R}=1 \forall s \in[0, L]$. As in [27, we use unit quaternions, i.e. normalized quadruples of real numbers $\mathbf{q}=\left(q_{1}, q_{2}, q_{3}, q_{4}\right)^{\top} \in \mathbb{R}^{4}:\|\mathbf{q}\|=1$, for the parameterization of frames resp. rotation matrices:

$$
\mathbf{q}:[0, L] \rightarrow S O(3) \leadsto \mathbf{R}(s)=\mathbf{R}(\mathbf{q}(s)),
$$

where

$$
\mathbf{R}(\mathbf{q})=\left(\begin{array}{ccc}
q_{1}^{2}-q_{2}^{2}-q_{3}^{2}+q_{4}^{2} & 2\left(q_{1} q_{2}-q_{3} q_{4}\right) & 2\left(q_{1} q_{3}+q_{2} q_{4}\right) \\
2\left(q_{1} q_{2}+q_{3} q_{4}\right) & -q_{1}^{2}+q_{2}^{2}-q_{3}^{2}+q_{4}^{2} & 2\left(q_{2} q_{3}-q_{1} q_{4}\right) \\
2\left(q_{1} q_{3}-q_{2} q_{4}\right) & 2\left(q_{2} q_{3}+q_{1} q_{4}\right) & -q_{1}^{2}-q_{2}^{2}+q_{3}^{2}+q_{4}^{2}
\end{array}\right) .
$$

Based on the centerline $\mathbf{r}(s)$ and rotation matrix $\mathbf{R}(s)$ associated with the frame, the kinematics of the Cosserat rod can be derived. Dropping the dependency on the arc-length parameter $s$ in the notation, the 
translatory strains are given as

$$
\varepsilon=\mathbf{R}^{\top} \mathbf{r}^{\prime}-\mathbf{e}_{3}=\mathbf{R}^{\top}\left(\mathbf{r}^{\prime}-\mathbf{g}_{3}\right),
$$

where $\mathbf{e}_{3}$ denotes the Cartesian basis vector $(0,0,1)^{\top}$. The rotational strains are defined in terms of the curvature vector of the rod:

$$
\boldsymbol{\kappa}=\left(\begin{array}{l}
\mathbf{g}_{2}^{\prime \top} \mathbf{g}_{3} \\
\mathbf{g}_{3}^{\prime \top} \mathbf{g}_{1} \\
\mathbf{g}_{1}^{\prime \top} \mathbf{g}_{2}
\end{array}\right) \quad \Leftrightarrow \quad[\boldsymbol{\kappa}]_{\times}=\mathbf{R}^{\prime \top} \mathbf{R}
$$

where $[\cdot]_{\times}$represents the skew-symmetric cross-product matrix.

Using these two nonlinear strain vectors and a linear elastic constitutive law, the translatory and rotational stresses can be computed as:

$$
\boldsymbol{\sigma}=\mathbf{C}\left(\varepsilon-\varepsilon_{0}\right), \quad \chi=\mathbf{D}\left(\boldsymbol{\kappa}-\boldsymbol{\kappa}_{0}\right)
$$

Here the terms $\varepsilon_{0}$ and $\boldsymbol{\kappa}_{0}$ represent strains in the initial configuration $\left(\mathbf{r}_{0}, \mathbf{R}_{0}\right)$ of a pre-stretched or precurved rod and the intrinsic material matrices $\mathbf{C}=\operatorname{diag}\left(k_{1} G A, k_{2} G A, E A\right), \mathbf{D}=\operatorname{diag}\left(E I_{1}, E I_{2}, G J\right)$ are used. These diagonal matrices depend on the material parameters elastic modulus $E$ and shear modulus $G=E /(2+2 \nu)$ with Poisson's ratio $\nu$, and on the geometrical cross-section parameters area $A$, second moments of area $I_{1}$ and $I_{2}$, torsion constant $J$, and shear correction factors $k_{1}$ and $k_{2}$.

The stress vectors defined in (7) are now rotated from the local into the global Euclidian coordinate frame, or in other words they are transformed from the spatial back to the material configuration. This results in the expressions for internal force and internal moment vectors:

$$
\mathbf{n}=\mathbf{R} \boldsymbol{\sigma}=\mathbf{R C}\left(\varepsilon-\varepsilon_{0}\right), \quad \mathbf{m}=\mathbf{R} \chi=\mathbf{R D}\left(\boldsymbol{\kappa}-\boldsymbol{\kappa}_{0}\right) .
$$

Finally, the governing equations of the mechanics of the Cosserat rod model are formulated in terms of equilibria of linear and angular momentum in the material or initial configuration:

$$
\begin{aligned}
\mathbf{n}^{\prime}+\hat{\mathbf{n}}=\mathbf{0} & \forall s \in(0, L), \\
\mathbf{m}^{\prime}+\mathbf{r}^{\prime} \times \mathbf{n}+\hat{\mathbf{m}}=\mathbf{0} & \forall s \in(0, L) .
\end{aligned}
$$

Here $\hat{\mathbf{n}}$ and $\hat{\mathbf{m}}$ are external distributed forces and moments. Furthermore, these differential equations have to be completed with appropriate boundary conditions at the two ends of the rod for $s=0$ and $s=L$. Fixed displacements $\overline{\mathbf{r}}$ and rotations $\overline{\mathbf{q}}$ are specified as Dirichlet boundary conditions $\mathbf{r}=\overline{\mathbf{r}}, \mathbf{q}=\overline{\mathbf{q}}$ at $s=0, L$ or forces $\overline{\mathbf{n}}$ and moments $\overline{\mathbf{m}}$ as Neumann boundary conditions $\mathbf{n}=\overline{\mathbf{n}}, \mathbf{m}=\overline{\mathbf{m}}$ at $s=0, L$. Additionally, a unit length constraint for quaternions must hold to complete the equilibrium equations: $\mathbf{q}^{\top} \mathbf{q}-1=0 \forall s \in[0, L]$.

\section{Isogeometric collocation method}

For the numerical discretization of the Cosserat rod model presented above we employ an isogeometric collocation method. The approach is based on the parameterization of the unknown fields $\mathbf{r}(s)$ and $\mathbf{q}(s)$ using spline functions and on the collocation of the equilibrium equations (9). This method was introduced and investigated in [27, and here we briefly review the basic approach.

\subsection{Spline parameterization of the rods}

The basis of any isogeometric method is the parameterization of geometry and unknowns using BSplines or Non-Uniform Rational B-Splines (NURBS), which are widely used in computer-aided design 6. 45. Definitions and properties of B-Splines and NURBS can be found in detail in 46. Here we just briefly introduce the main terminology associated with splines.

B-Splines are piecewise polynomial functions and NURBS piecewise rational functions of degree $p$ and order $p+1$. With $N_{i}(t): \Omega_{0} \rightarrow[0,1], i=1, \ldots, n$ we denote the spline (B-Spline or NURBS) basis functions, 


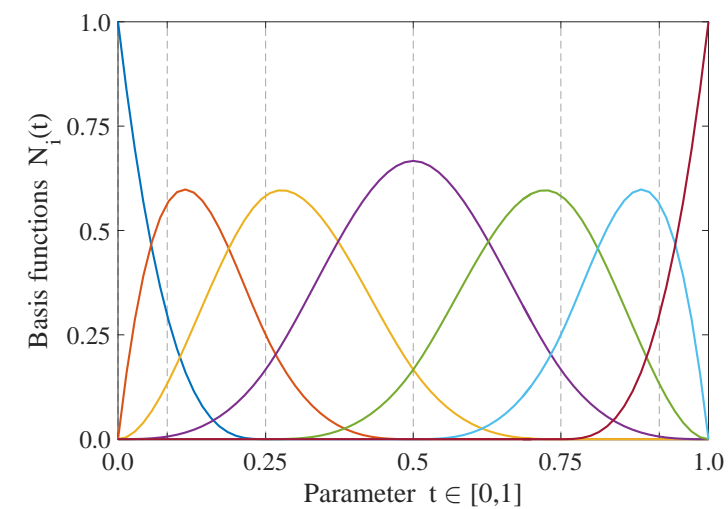

(a) B-Spline basis functions

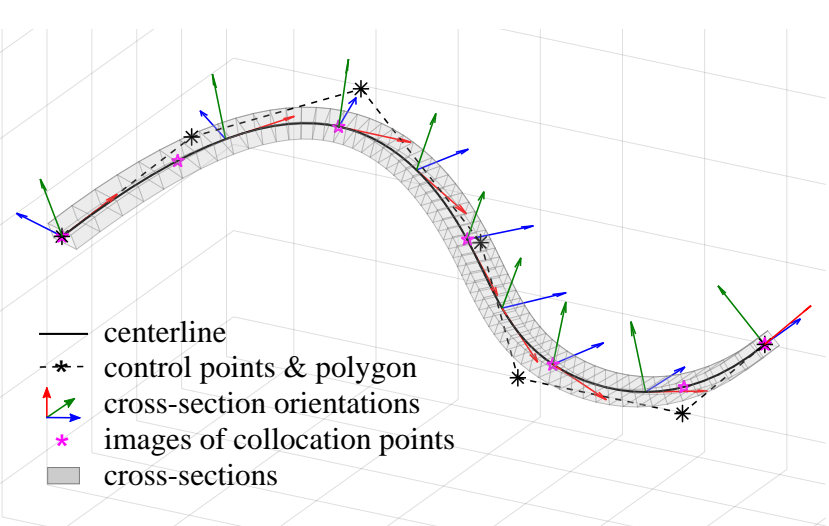

(b) Isogeometric parameterization of rod with B-Spline curve

Figure 2: Isogeometric rod parameterization; cubic B-Spline basis functions as shown in (a) with $p=3, m=11, n=7, \Xi=$ $\left\{0,0,0,0, \frac{1}{4}, \frac{1}{2}, \frac{3}{4}, 1,1,1,1\right\}$ are used for the isogeometric parameterization of the Cosserat rod in (b) with centerline and rotation quaternions as B-Spline curves 27 .

which are defined on the parameter domain $\Omega_{0}=\left[\xi_{1}, \xi_{m}\right] \subset \mathbb{R}$ using a knot vector $\Xi=\left\{\xi_{1}, \ldots, \xi_{m}\right\}$ with $m=n+p+1$, i.e. a non-decreasing sequence of knots $\xi_{i} \in \mathbb{R}(i=1, \ldots, m), \xi_{i} \leq \xi_{i+1}(i=1, \ldots, m-1)$. For two distinct knots $\xi_{i} \neq \xi_{i+1}$ the half-open interval $\left[\xi_{i}, \xi_{i+1}\right)$ is called the $i$-th knot span or element and the total number of nonzero knot spans or elements in $\Xi$ is denoted by $\ell$. Typically, only open knot vectors are used in IGA, which means that the first and last knots have multiplicity $p+1$, with inner knots of multiplicity $1 \leq k \leq p$.

The geometry description of a Cosserat rod can now be expressed using spline curves for the initial centerline $\mathbf{r}_{0}$ and rotation quaternions $\mathbf{q}_{0}$ :

$$
\mathbf{r}_{0}: \Omega_{0} \rightarrow \mathbb{R}^{3}, \mathbf{r}_{0}(t)=\sum_{i=1}^{n} N_{i}(t) \mathbf{r}_{0, i} \quad \mathbf{q}_{0}: \Omega_{0} \rightarrow \mathbb{R}^{4}, \mathbf{q}_{0}(t)=\sum_{i=1}^{n} N_{i}(t) \mathbf{q}_{0, i},\left\|\mathbf{q}_{0}(t)\right\|=1
$$

with control points $\left\{\mathbf{r}_{0, i}\right\}_{i=1, \ldots, n}, \mathbf{r}_{0, i} \in \mathbb{R}^{3}$ and $\left\{\mathbf{q}_{0, i}\right\}_{i=1, \ldots, n}, \mathbf{q}_{0, i} \in \mathbb{R}^{4}$.

For illustration, the parameterization of a rod using cubic B-Spline basis functions $(p=3)$ with $n=7$ control points and $\ell=4$ elements in the knot vector $\Xi=\left\{0,0,0,0, \frac{1}{4}, \frac{1}{2}, \frac{3}{4}, 1,1,1,1\right\}$ is shown in Fig. 2 . Figure 2a shows the basis functions and Fig. $2 \mathrm{~b}$ the rod itself in terms of its centerline curve and crosssection frames.

Since the centerline is now parameterized as a spline curve $\mathbf{r}_{0}(t): \Omega_{0} \rightarrow \mathbb{R}^{3}$ with an arbitrary domain of parameterization $\Omega_{0} \subset \mathbb{R}$, it is in general not arc-length parameterized. Thus the derivatives of any vector field $t \rightarrow \mathbf{y}(t):[0,1] \rightarrow \mathbb{R}^{d}$ required for evaluation of the Cosserat rod model need to be converted to arc-length parameterization using:

$$
\mathbf{y}^{\prime}=\frac{d \mathbf{y}}{d s}=\frac{d \mathbf{y}}{d t} \frac{d t}{d s}=\dot{\mathbf{y}}\left(\frac{d s}{d t}\right)^{-1}=\dot{\mathbf{y}} \frac{1}{\left\|\dot{\mathbf{r}}_{0}(t)\right\|}=\frac{1}{J} \dot{\mathbf{y}}
$$

with $\dot{\mathbf{y}}:=d \mathbf{y} / d t$ and $J(t):=\left\|\dot{\mathbf{r}}_{0}(t)\right\|$.

\subsection{Collocation of strong equilibrium equations}

As in isoparametric finite elements, the two unknown solution fields, the centerline position $\mathbf{r}$ and rotation quaternion $\mathbf{q}$ in the current/deformed configuration, are now discretized as spline curves $\mathbf{r}_{h}$ and $\mathbf{q}_{h}-$ just 
like their initial counterparts $\mathbf{r}_{0}$ and $\mathbf{q}_{0}$ in $10 p$ :

$$
\mathbf{r}_{h}: \Omega_{0} \rightarrow \mathbb{R}^{3}, \mathbf{r}_{h}(t)=\sum_{i=1}^{n} N_{i}(t) \mathbf{r}_{i}, \quad \mathbf{q}_{h}: \Omega_{0} \rightarrow \mathbb{R}^{4}, \mathbf{q}_{h}(t)=\sum_{i=1}^{n} N_{i}(t) \mathbf{q}_{i},\left\|\mathbf{q}_{h}(t)\right\|=1 .
$$

Here, the basis functions $N_{i}$ refer to either the same or $p$-/ $h$ - $/ k$-refined versions of the ones in 100 and the control points $\mathbf{r}_{i} \in \mathbb{R}^{3}$ and $\mathbf{q}_{i} \in \mathbb{R}^{4}$ are stacked into two vectors $\overrightarrow{\mathbf{r}}=\left(\mathbf{r}_{i}\right)_{i=1, \ldots, n} \in \mathbb{R}^{3 n}$ and $\overrightarrow{\mathbf{q}}=\left(\mathbf{q}_{i}\right)_{i=1, \ldots, n} \in$ $\mathbb{R}^{4 n}$.

Now collocation of the strong form of the equilibrium equations of the Cosserat rod is applied, i.e. the discretized unknowns from (12) are used to evaluate the forces $\mathbf{n}_{h}$ and moments $\mathbf{m}_{h}$ of the discretized model at a set of collocation points $\left\{\tau_{i}\right\}_{i=1, \ldots, n}$ and then substituted into the balance equations $(9)$ and the quaternion normalization condition:

$$
\begin{aligned}
\mathbf{f}_{\mathbf{n}}\left(\tau_{i}\right) & = & \mathbf{n}_{h}^{\prime}\left(\tau_{i}\right)+\hat{\mathbf{n}}\left(\tau_{i}\right) & =\mathbf{0}, \\
\mathbf{f}_{\mathbf{m}}\left(\tau_{i}\right) & = & \mathbf{m}_{h}^{\prime}\left(\tau_{i}\right)+\mathbf{r}_{h}^{\prime}\left(\tau_{i}\right) \times \mathbf{n}_{h}\left(\tau_{i}\right)+\hat{\mathbf{m}}\left(\tau_{i}\right) & =\mathbf{0}, \\
\mathbf{f}_{\mathbf{q}}\left(\tau_{i}\right) & = & \mathbf{q}_{h}\left(\tau_{i}\right)^{\top} \mathbf{q}_{h}\left(\tau_{i}\right)-1 & =0 .
\end{aligned}
$$

At the boundary, i.e. for $i=1$ and $i=n$, the above-mentioned equations are replaced with the evaluations of the boundary conditions. In order to guarantee the stability of the method, the collocation points are chosen as the Greville abscissae of the spline knot vector [15], which are defined as:

$$
\tau_{i}=\frac{\xi_{i+1}+\ldots+\xi_{i+p}}{p}, i=1, \ldots, n .
$$

With (13) we have defined a nonlinear system of $7 n$ equations for the $7 n$ unknowns, i.e. the control point vectors $\overrightarrow{\mathbf{r}}$ for $\mathbf{r}_{h}$ and $\overrightarrow{\mathbf{q}}$ for $\mathbf{q}_{h}$, which we can write as:

$$
\mathbf{f}: \mathbb{R}^{3 n} \times \mathbb{R}^{4 n} \rightarrow \mathbb{R}^{7 n}: \quad \mathbf{f}(\overrightarrow{\mathbf{r}}, \overrightarrow{\mathbf{q}})=\left(\begin{array}{c}
\mathbf{f}_{\mathrm{n}}\left(\tau_{i}\right) \\
\mathbf{f}_{\mathrm{m}}\left(\tau_{i}\right) \\
\mathbf{f}_{\mathbf{q}}\left(\tau_{i}\right)
\end{array}\right)_{i=1, \ldots, n}(\overrightarrow{\mathbf{r}}, \overrightarrow{\mathbf{q}})=\mathbf{0}
$$

This nonlinear system is then solved with Newton's method, which requires also the evaluation of the stiffness matrix $\mathbf{K}(\overrightarrow{\mathbf{r}}, \overrightarrow{\mathbf{q}})=d \mathbf{f} / d(\overrightarrow{\mathbf{r}}, \overrightarrow{\mathbf{q}})$ for linearization, see 27, for details.

In [27] this isogeometric collocation formulation was also extended to a mixed method that resolves shear locking for thin rods, and furthermore to rod structures with several interconnected rods.

\section{Contact formulation}

Having introduced the Cosserat rod model and its numerical discretization in the previous sections, we now establish a contact formulation for frictionless rod-to-rod contact within the isogeometric collocation framework. We assume a circular cross-sectional shape. Within contact formulations two main tasks can be usually distinguished, namely the geometrical contact detection and the mechanical contact model and force calculation [47. In the context of isogeometric collocation, a major challenge is the application of the contact forces as point forces. In the following, we describe our approaches to these sub-problems in detail and outline the overall contact algorithm.

\subsection{Geometric contact detection}

The main tasks of the geometric part of the contact algorithm are to find possible locations where contact between two rods of an assembly with $n_{R}$ rods might occur, to check the contact status (active or inactive) at these locations and to compute the normal direction to be used for the later computation of the contact forces in case of active contact. Since the rod model is based only on the parameterization of the centerline and the cross-section orientation, a representation of the actual outer surface of a rod is not 
directly available. As in other works [1, we thus divide the search for contact points into three major steps, which are described as follows.

\subsubsection{Coarse-level contact candidate search}

First, the parametric space of each $\operatorname{rod} \mathbf{r}^{I}$ is discretized into $n_{c}^{I}$ contact candidate parameters $\eta_{i}^{I} \in \Omega_{0}(i=$ $\left.1, \ldots, n_{c}^{I}\right)$ and then the current centerline positions $\mathbf{r}^{I}\left(\eta_{i}^{I}\right)$ are evaluated at these locations. These candidate parameters may be chosen to be the same as the collocation points, i.e. $n_{c}^{I}=n^{I}$ and $\eta_{i}^{I}=\tau_{i}^{I}$, see (14). However, since the collocation points are concentrated at the end of the parameter interval, equidistantly spaced points are preferable, i.e. $\eta_{i}^{I}=\frac{i-1}{n_{c}^{I}-1}$ for $\Omega_{0}^{I}=[0,1]$.

Now a coarse level search is performed by finding all pairs of contact candidates of rods and parameters $(I, i ; J, j)$ for which

$$
\left\|\mathbf{r}^{I}\left(\eta_{i}^{I}\right)-\mathbf{r}^{J}\left(\eta_{j}^{J}\right)\right\|<\varepsilon,
$$

where the superscripts ${ }^{I}$ and ${ }^{J}$ denote two distinct rods from the assembly, i.e. $I, J \in\left\{1, \ldots, n_{R}\right\}, i \in$ $\left\{1, \ldots, n_{c}^{I}\right\}, j \in\left\{1, \ldots, n_{c}^{J}\right\}$, and $\varepsilon$ is a threshold which should be chosen large enough to ensure that no possible contact locations are missed, but small enough to consider not too many unnecessary locations where no contact will occur. Typically we chose $2\left(r^{I}+r^{J}\right) \leq \varepsilon \leq 4\left(r^{I}+r^{J}\right)$, where $r^{I}$ are the cross-section radii. This procedure is illustrated in Fig. 3a.

For a computationally efficient implementation of this search, which requires comparison of many evaluation points $\mathbf{r}^{I}\left(\eta_{i}^{I}\right)$ and $\mathbf{r}^{J}\left(\eta_{j}^{J}\right)$ with each other, nearest neigbhbour algorithms and data structures such as bounding volume hierarchies can be considered [48. If self-contact within a rod is to be included in the search, it has to be carried out for $J \geq I$ and for $I=J$ the parameter indices are restricted to $\eta_{j}^{I}>\eta_{i}^{I}+\delta_{s}$, where $\delta_{s}$ represents an offset to avoid finding unncessary and unphysical contact locations along the centerline. Otherwise the search can be restricted to $J>I$.

\subsubsection{Refined search and initialization}

The second step is the continuous search for the parameters of the exact centerline points that are closest to each other within the neighbourhood of the candidate parameters of a contact candidate pair $(I, i ; J, j)$. Mathematically, this means that we are looking for

$$
\left(\hat{\eta}_{i}^{I}, \hat{\eta}_{j}^{J}\right)=\underset{\substack{\eta_{i}^{I-} \leq \hat{\eta}_{i}^{I} \leq \eta_{i}^{I+} \\ \eta_{j}^{J-} \leq \hat{\eta}_{j}^{J} \leq \eta_{j}^{J+}}}{\arg \min }\left\|\mathbf{r}^{I}\left(\hat{\eta}_{i}^{I}\right)-\mathbf{r}^{J}\left(\hat{\eta}_{j}^{J}\right)\right\|,
$$

where we restrict the search between $\eta_{i}^{I-}=\frac{1}{2}\left(\eta_{i-1}^{I}+\eta_{i}^{I}\right)$ and $\eta_{i}^{I+}=\frac{1}{2}\left(\eta_{i+1}^{I}+\eta_{i}^{I}\right)$. Assuming that the objective function is convex within the given small parameter ranges, the minimization problem can be solved by using Newton-Raphson's method. This step is also illustrated in Fig. 3b.

Now that we have determined the parameters of the closest centerline points, we can compute the normalized direction vector between the two centerline points:

$$
\mathbf{d}_{c}=\frac{\mathbf{r}^{J}\left(\hat{\eta}_{j}\right)-\mathbf{r}^{I}\left(\hat{\eta}_{i}\right)}{\left\|\mathbf{r}^{J}\left(\hat{\eta}_{j}\right)-\mathbf{r}^{I}\left(\hat{\eta}_{i}\right)\right\|} .
$$

Based on this direction vector and the tangents of the centerline curve, the normal contact direction, which predicts the direction in which contact forces (in case of active contact) will act on both rods, is computed:

$$
\mathbf{n}_{c}=\frac{\left(\mathbf{t}_{c} \times \mathbf{d}_{c}\right) \times \mathbf{t}_{c}}{\left\|\left(\mathbf{t}_{c} \times \mathbf{d}_{c}\right) \times \mathbf{t}_{c}\right\|} .
$$

Here we have used the normalized direction vector $\mathbf{d}_{c}$, as well as the averaged unit tangent vector:

$$
\mathbf{t}_{c}=\frac{\mathbf{r}^{I^{\prime}}\left(\hat{\eta}_{i}\right)+\mathbf{r}^{J^{\prime}}\left(\hat{\eta}_{j}\right)}{\left\|\mathbf{r}^{I^{\prime}}\left(\hat{\eta}_{i}\right)+\mathbf{r}^{J^{\prime}}\left(\hat{\eta}_{j}\right)\right\|} .
$$




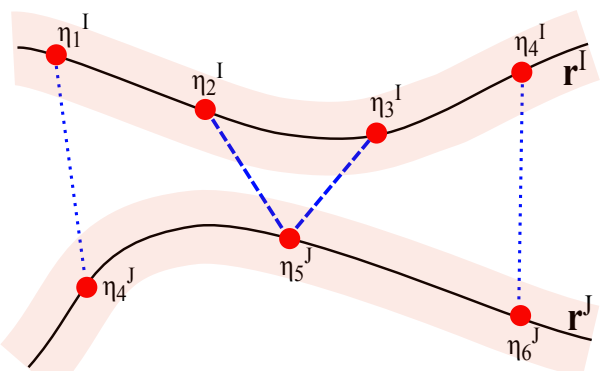

(a) Coarse level search, where only the dashed pairs are considered as contact candidates, since $\left\|\mathbf{r}^{I}\left(\eta_{i}^{I}\right)-\mathbf{r}^{J}\left(\eta_{j}^{J}\right)\right\|<\varepsilon$

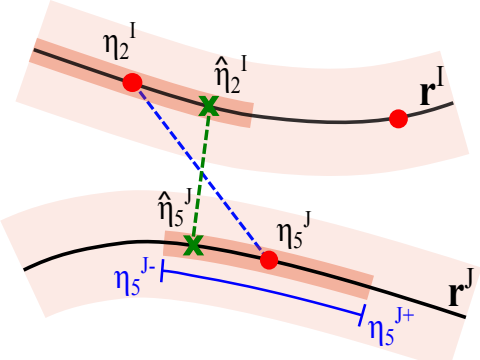

(b) Continuous search for closest point parameters $\left(\hat{\eta}_{i}^{I}, \hat{\eta}_{j}^{J}\right)$ of each candidate pair $(I, i ; J, j)$

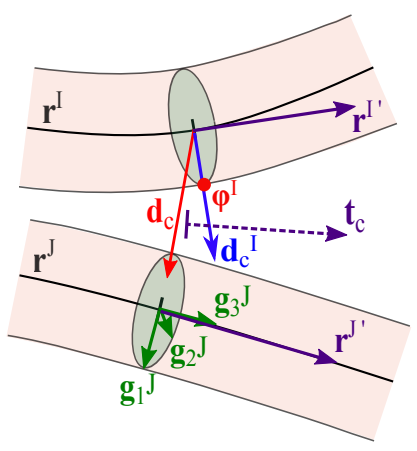

(c) The surface parameters $\phi^{I}$ are computed based on $\mathbf{d}_{c}$ and its projection $\mathbf{d}_{c}^{I}$

Figure 3: Geometric contact detection. The three main steps of the search for contact candidate points are illustrated.

The convoluted expression in $\sqrt{19}$ is used to circumvent problems with (almost) parallel rods, where the simpler definition

$$
\mathbf{n}_{c}=\frac{\mathbf{r}^{I^{\prime}}\left(\hat{\eta}_{i}\right) \times \mathbf{r}^{J^{\prime}}\left(\hat{\eta}_{j}\right)}{\left\|\mathbf{r}^{I^{\prime}}\left(\hat{\eta}_{i}\right) \times \mathbf{r}^{J^{\prime}}\left(\hat{\eta}_{j}\right)\right\|}
$$

would fail, see [1]. Fig. $3 \mathrm{c}$ shows the direction vector $\mathbf{d}_{c}$ and averaged tangent $\mathbf{t}_{c}$, but the normal $\mathbf{n}_{c}$ is not visualized since it roughly coincides with $\mathbf{d}_{c}$.

\subsubsection{Gap function evaluation}

We proceed to determine the points on the outer surface of each rod that are likely to come into contact or penetrate each other, which will allow us to calculate the gap or amount of penetration that occurs, see [1] and Fig. $3 \mathrm{c}$ for illustration.

First, the normalized direction vector $\mathbf{d}_{c}$ is projected onto the cross-section planes of both rods:

$$
\mathbf{d}_{c}^{I}=\mathbf{d}_{c}-\left(\mathbf{d}_{c}^{\top} \mathbf{g}_{3}^{I}\right) \mathbf{g}_{3}^{I}, \quad \mathbf{d}_{c}^{J}=\mathbf{d}_{c}-\left(\mathbf{d}_{c}^{\top} \mathbf{g}_{3}^{J}\right) \mathbf{g}_{3}^{J} .
$$

Here $\mathbf{g}_{3}^{I}$ is the third column of the cross-section orientation matrix $\mathbf{R}^{I}\left(\hat{\eta}_{i}\right)$, which is orthogonal to the other two unit vectors $\mathbf{g}_{1}^{I}$ and $\mathbf{g}_{2}^{I}$ that span the cross-sectional plane of $\operatorname{rod} I$ at $\hat{\eta}_{i}$, see (2). Then the crosssectional parameters $\left(\phi_{1}^{I}, \phi_{2}^{I}\right)$ of the surface point which is candidate to contact can be computed for each rod by solving the following two systems of equations:

$$
\begin{aligned}
\phi_{1}^{I} \mathbf{g}_{1}^{I}+\phi_{2}^{I} \mathbf{g}_{2}^{I} & =\alpha^{I} \mathbf{d}_{c}^{I}, & \phi_{1}^{J} \mathbf{g}_{1}^{J}+\phi_{2}^{J} \mathbf{g}_{2}^{J}= & \alpha^{J} \mathbf{d}_{c}^{J}, \\
\phi_{1}^{I^{2}}+\phi_{2}^{I^{2}} & =r^{I^{2}}, & \phi_{1}^{J^{2}}+\phi_{2}^{J^{2}} & =r^{J^{2}}
\end{aligned}
$$

where $\alpha^{I}>0$ and $\alpha^{J}<0$, see [1].

Using the refined centerline parameters $\hat{\eta}_{i}$ and cross-sectional parameters $\left(\phi_{1}^{I}, \phi_{2}^{I}\right)$, we can evaluate the physical locations of both contact candidate surface points:

$$
\mathbf{x}_{i}^{I}\left(\phi_{i}^{I}\right)=\mathbf{r}^{I}\left(\hat{\eta}_{i}\right)+\phi_{1}^{I} \mathbf{g}_{1}^{I}+\phi_{2}^{I} \mathbf{g}_{2}^{I}, \quad \quad \mathbf{x}_{j}^{J}\left(\phi_{j}^{J}\right)=\mathbf{r}^{J}\left(\hat{\eta}_{j}\right)+\phi_{1}^{J} \mathbf{g}_{1}^{J}+\phi_{2}^{J} \mathbf{g}_{2}^{J}
$$

where $\phi_{i}^{I}=\left(\hat{\eta}_{i} ; \phi_{1}^{I}, \phi_{2}^{I}\right)$ and $\phi_{j}^{J}=\left(\hat{\eta}_{j} ; \phi_{1}^{J}, \phi_{2}^{J}\right)$.

Based on the evaluation of the surface points $\mathbf{x}_{i}^{I}$ and $\mathbf{x}_{i}^{J}$ and the normal direction $\mathbf{n}_{c}$, the gap function, which measures the distance between both surface points along the normal contact direction, is computed:

$$
g=\left(\mathbf{x}_{j}^{J}\left(\phi_{j}^{J}\right)-\mathbf{x}_{i}^{I}\left(\phi_{i}^{I}\right)\right)^{\top} \mathbf{n}_{c}
$$




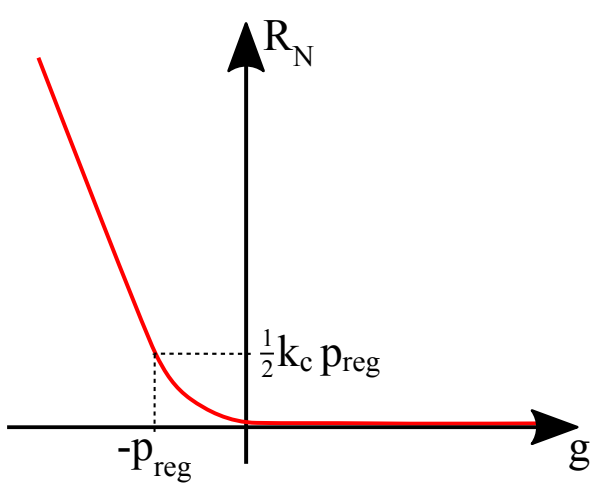

Figure 4: Dependency of penalty contact force $R_{N}$ on gap function value $g$

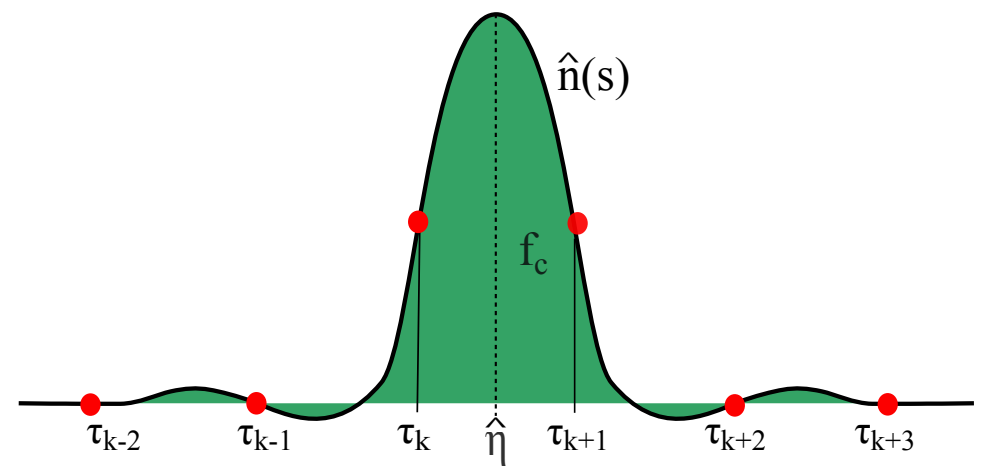

Figure 5: The distributed load function $\hat{\mathbf{n}}(s)$ interpolates the point load at the collocation points $\tau_{k}$, has its peak at $\hat{\eta}$ and its integral is equal to $\mathbf{f}_{c}$

For $g>0$ the rods are separated from each other, for $g=0$ they are exactly in contact, and for $g<0$ they are already penetrating each other.

\subsection{Contact force calculation}

Having determined the major geometric quantities of the contact problem, namely the normal contact direction in 19 and the gap function value 25 , now the mechanical part of the contact treatment can take place for every contact candidate pair $(I, i ; J, j)$.

The value of the gap function is used to compute a penalty contact force, which stems from the enforcement of the non-penetration constraints with the penalty method:

$$
R_{N}= \begin{cases}0 & , 0 \leq g, \\ \frac{k_{c}}{2 p_{r e g}} g^{2} & ,-p_{r e g} \leq g<0, \\ -k_{c}\left(g+\frac{p_{r e g}}{2}\right) & , g<-p_{\text {reg }} .\end{cases}
$$

As in [1, we have chosen here an expression which is continuously differentiable at $g=0$. It depends on the penalty factor $k_{c} \gg 0$ and the regularization parameter $p_{\text {reg }}>0$, see Fig. 4 .

The penalty contact force and normal direction are then used to assign the contact reaction forces to both rods:

$$
\mathbf{f}_{c}^{I}\left(\hat{\eta}_{i}\right)=-R_{N} \mathbf{n}_{c}, \quad \mathbf{f}_{c}^{J}\left(\hat{\eta}_{j}\right)=R_{N} \mathbf{n}_{c} .
$$

These forces act as oppositely directed, external point loads at the centerline parameters $\hat{\eta}_{i}$ and $\hat{\eta}_{j}$ within the balance equations of linear momentum $\sqrt{8}$ for $\operatorname{rod} I$ and $\operatorname{rod} J$.

For frictionless contact, the penalization forces represent the mechanical reaction forces acting at the contact interface of two contacting rods with an ideal gap $g=0$. Thus they must be non-zero in the case of contact, which means that only a gap function value $g<0$ leads to the correct contact forces. As a consequence, a contact penalty method such as the one presented here always leads to a small penetration of contacting rods. The amount of penetration can be controlled by the two parameters $k_{c}$ and $p_{\text {reg }}$ in (26).

\subsection{Point forces in isogeometric collocation}

Having determined the contact reaction forces $\mathbf{f}_{c}$ and their parameter locations $\hat{\eta}$ (dropping now the indices $I$ and $i$ ), we now need to assign these forces to the discretized rod model.

If the external force acts directly on a boundary collocation point, i.e. $\hat{\eta}=\tau_{1}$ or $\hat{\eta}=\tau_{n}$, we can directly prescribe it using the Neumann boundary condition $\mathbf{n}(\hat{\eta})=\overline{\mathbf{n}}(\hat{\eta}) \simeq \mathbf{f}_{c}$. However, within the isogeometric collocation approach, there are two major challenges when point forces have to be applied in the interior, see (13): (a) the equilibrium equations are only evaluated at discrete collocation points $\tau_{i}$, which in general do not correspond to the refined contact point parameters $\hat{\eta}$; and (b) the external forces in the balance 
equation have to be specified as line loads: $\mathbf{n}_{h}^{\prime}\left(\tau_{i}\right)=-\hat{\mathbf{n}}\left(\tau_{i}\right)$. This issue was already briefly addressed for collocation of the Kirchhoff plate model using T-Splines in [49, but restricted to point forces acting directly at a collocation point. Here, we pick up the idea and generalize it to an arbitrary parameter of attack of the point force.

As mentioned above, at interior collocation points only line loads can be prescribed, i.e. $\mathbf{n}_{h}^{\prime}\left(\tau_{i}\right)=-\hat{\mathbf{n}}\left(\tau_{i}\right)$ when $1<i<n$, see (13). Since the contact force is only acting as a pointwise Dirac delta function, it is not differentiable and $\mathbf{f}_{c}{ }^{\prime}\left(\tau_{i}\right) \simeq \hat{\mathbf{n}}\left(\tau_{i}\right)$ is not well defined. Thus, we need to regard $\mathbf{f}_{c}$ as the resultant of a distributed line load function $\hat{\mathbf{n}}:[0, L] \rightarrow \mathbb{R}^{3}$. We define the 2-point method for point forces such that for the distributed load it shall hold that:

- it is zero at all collocation points except the two nearest ones to $\hat{\eta}$, i.e. with $\tau_{k}<\hat{\eta} \leq \tau_{k+1}$ it is:

$$
\hat{\mathbf{n}}\left(\tau_{i}\right)= \begin{cases}\lambda \frac{\tau_{k+1}-\hat{\eta}}{\tau_{k+1}-\tau_{k}} \mathbf{f}_{c} & \text { if } i=k, \\ \lambda \frac{\hat{\eta}-\tau_{k}}{\tau_{k+1}-\tau_{k}} \mathbf{f}_{c} & \text { if } i=k+1 \\ \mathbf{0} & \text { else, }\end{cases}
$$

for $\lambda>0$,

- its integral is equal to the value of the point force:

$$
\int_{0}^{L} \hat{\mathbf{n}}(s) d s=\mathbf{f}_{c},
$$

which determines the factor $\lambda$ above.

With these two conditions, we can determine the discretized line load as an interpolatory spline curve $\hat{\mathbf{n}}_{h}(t)=\sum_{i=1}^{n} N_{i}(t) \mathbf{n}_{i}$ using the same basis functions from $\left[12\right.$, see Fig. 5 . In the case $\hat{\eta}=\tau_{k+1}$ this corresponds to the formulation used in 49 .

Similarly, we can also introduce the definition of an alternative 4-point method such that the distributed line load function $\hat{\mathbf{n}}:[0, L] \rightarrow \mathbb{R}^{3}$ :

- is zero at all collocation points except the four nearest ones to $\hat{\eta}$, i.e. with $\tau_{k}<\hat{\eta} \leq \tau_{k+1}$ it is:

$$
\hat{\mathbf{n}}\left(\tau_{i}\right)= \begin{cases}\lambda \frac{\tau_{k+1}-\hat{\eta}}{\tau_{k}-\tau_{k-1}} \mathbf{f}_{c} & \text { if } i=k-1 \\ \lambda\left(\frac{1}{2}+\frac{\tau_{k+1}-\hat{\eta}}{\tau_{k+1}-\tau_{k-1}} \mathbf{f}_{c}\right) & \text { if } i=k, \\ \lambda\left(\frac{1}{2}+\frac{\hat{\eta}-\tau_{k}}{\tau_{k+2}-\tau_{k}} \mathbf{f}_{c}\right) & \text { if } i=k+1 \\ \lambda \frac{\hat{\eta}-\tau_{k}}{\tau_{k+2}-\tau_{k+1}} \mathbf{f}_{c} & \text { if } i=k+2 \\ \mathbf{0} & \text { else, }\end{cases}
$$

for $\lambda>0$,

- its integral is equal to the value of the point force:

$$
\int_{0}^{L} \hat{\mathbf{n}}(s) d s=\mathbf{f}_{c},
$$

which again determines the factor $\lambda$ above.

In this way the point force is distributed or "smeared" over a larger range of collocation points compared to the 2-point version.

In general, a drawback of both methods is that they require the integrals in either (29) or (31) to be computed for every contact candidate pair, which has to be done numerically, e.g. using Gauss integration. This amounts to a relatively large computational effort, especially when many contact points exist, and is unsatisfactory in the context of a collocation method, which attempts to avoid integration. 
However, from our numerical experiments (see Sect. 5.2. Fig. 11b) we can see that the 2-point method leads basically to:

$$
\begin{aligned}
\hat{\mathbf{n}}\left(\tau_{k}\right) & =\frac{1}{J(\hat{\eta})\left(\tau_{k+1}-\tau_{k}\right)} \frac{\tau_{k+1}-\hat{\eta}_{i}}{\tau_{k+1}-\tau_{k}} \mathbf{f}_{c}, \\
\hat{\mathbf{n}}\left(\tau_{k+1}\right) & =\frac{1}{J(\hat{\eta})\left(\tau_{k+1}-\tau_{k}\right)} \frac{\hat{\eta}_{i}-\tau_{k}}{\tau_{k+1}-\tau_{k}} \mathbf{f}_{c},
\end{aligned}
$$

if the collocation point $\tau_{i}$ is not near the boundary and the parameterization is linear, i.e. $J=$ const. This means that:

$$
\begin{aligned}
\frac{1}{\lambda} & \approx J(\hat{\eta})\left(\tau_{k+1}-\tau_{k}\right), \\
\Rightarrow \quad \lambda & \approx \frac{1}{J(\hat{\eta})\left(\tau_{k+1}-\tau_{k}\right)} .
\end{aligned}
$$

The resulting approximation of $\lambda$ can be used in the 2-point method instead of the actual value of $\lambda$, avoiding the computationally expensive integration approach presented above. In the case of applying contact penalty forces, this approximation for $\lambda$ and usage of the "2-point, $J \Delta \tau$-method" are in general justified, if the error made is similar or smaller than all other errors introduced in the contact discretization, i.e. the error coming from the gap and choice of penalty factor, the isogeometric discretization error, etc. As the numerical results in Sect. 5.2 show, this is typically the case, especially since the 2-point and 4-point method with exact $\lambda$ also only provide an approximate application of point forces in collocation.

\subsection{Overview of contact algorithm and implementation}

Having addressed the individual parts of the rod-to-rod contact formulation in the preceding subsections, we now recap the contact algorithm and explain further details regarding the implementation in the context of isogeometric collocation of the Cosserat rod model.

In order to facilitate convergence, nonlinear static analyses are very often subdivided into a number of load steps, where the external forces are gradually increased. Within each load step, the contact points need to be determined and the nonlinear system $\mathbf{f}(\overrightarrow{\mathbf{r}}, \overrightarrow{\mathbf{q}})=\mathbf{0}$ is solved using Newton's method. Then the converged result of a load step serves as input for the next. The integration of the contact formulation into this framework is shown in Algorithm 1 .

The first part of the geometric contact treatment is placed at the beginning of a load step, where the contact candidate pairs are established using the coarse-level contact detection (see Sect. 4.1.1). The further steps are then conducted within a separate contact refinement loop. For all contact candidates, the refined search for the exact closest centerline points and the initialization of the normal direction vector and crosssection surface parameters are carried out (Sect. 4.1.2 and Sect.4.1.3). These are kept constant throughout the following Newton iteration process.

During each iteration of the Newton solver, the degree of penetration, i.e. the gap function, is evaluated based on the current deformation (see Sect. 4.1.3) and the resultant contact force for each contact pair is computed (see Sect. 4.2). After the usual assembly of the nonlinear system $\mathbf{f}(\overrightarrow{\mathbf{r}}, \overrightarrow{\mathbf{q}})=\mathbf{0}$ based on the discretizations of the individual rods 15 , the contact point loads are applied to the respective rows of $\mathbf{f}$ and their derivatives to $\mathbf{K}$.

These nested loops, both the Newton iteration and the contact refinement loop, can each be iterated until convergence, or be restricted to one of the following edge cases. If only a single iteration of the contact refinement loop is carried out, the refined contact search is only conducted once and the Newton iteration is then repeated until the desired convergence. In the second case, each Newton step is carried out only once while the contact refinement loop is repeated until convergence. This effectively updates the refined contact parameters and normal direction at each Newton iteration, as opposed to the first case where they remain constant throughout the Newton loop. Furthermore, in the second case it is important that the load step is small enough such that the normal contact direction does not flip during the iteration, which could happen when the centerline of one rod overshoots the centerline of the other. 


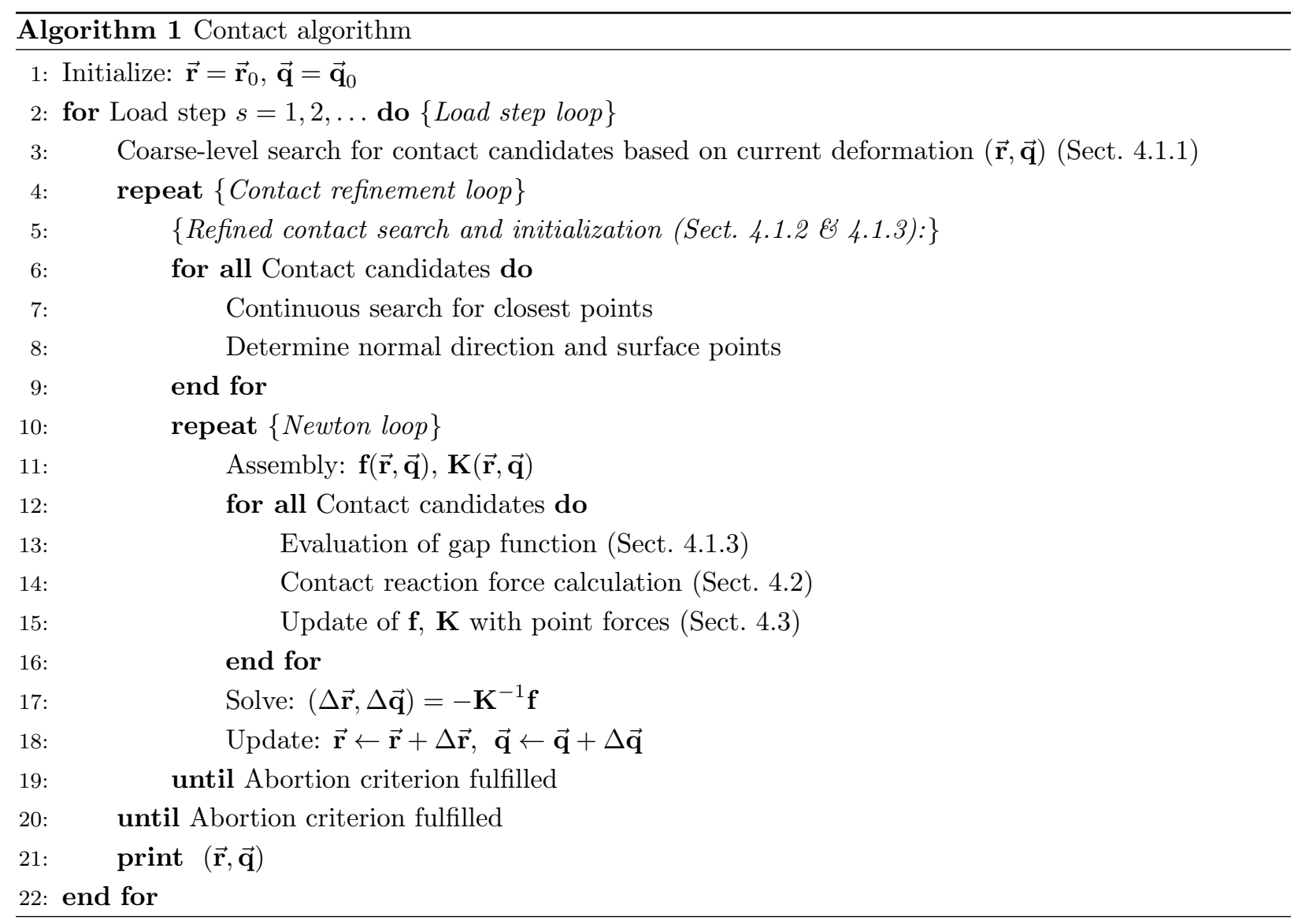

\section{Numerical applications}

In this section we verify the implementation of our contact algorithm within the isogeometric collocation framework and apply it to the numerical solution of complex frictionless rod-to-rod contact problems.

\subsection{Evaluation of point force method}

First, we study the accuracy and convergence of the point force application methods outlined in Sect. 4.3 using two verification examples:

- The "cantilever beam" in Fig. 6 is clamped at its left end $\left(\mathbf{r}(0)=\mathbf{r}_{0}(0), \mathbf{q}(0)=\mathbf{q}_{0}(0)\right)$ and free at the right end. The "half beam" version has a length of $L=0.5$ with parameter domain $\Omega_{0}=(0,0.5)$ and deforms under a prescribed common boundary force of $\overline{\mathbf{n}}(0.5)=\mathbf{f}=(0,0,-0.5)^{\top}$. The "full beam" has a length of $L=1.0$ with $\Omega_{0}=(0,1.0)$ and deforms under an internal point load $\mathbf{f}_{c}(0.5)=\mathbf{f}=$ $(0,0,-0.5)^{\top}$.

- The "double cantilever beam" in Fig. 7 is clamped at its left end $\left(\mathbf{r}(0)=\mathbf{r}_{0}(0), \mathbf{q}(0)=\mathbf{q}_{0}(0)\right)$ and undergoes symmetric deformation. The "half beam" version has a length of $L=0.5$ with parameter domain $\Omega_{0}=(0,0.5)$, fixed rotation on the right end $\left(\mathbf{q}(0.5)=\mathbf{q}_{0}(0.5)\right)$ and deforms under a prescribed common boundary force of $\overline{\mathbf{n}}(0.5)=\mathbf{f}=(0,0,-2)^{\top}$. The "full beam" has a length of $L=1.0$ with $\Omega_{0}=(0,1.0)$, a ruler support with fixed rotation and fixed $y$ - and $z$-deformation, and it deforms under an internal point load $\mathbf{f}_{c}(0.5)=2 \mathbf{f}=(0,0,-4)^{\top}$.

Due to the chosen boundary conditions and loads, for both examples the deformation of the "full beams" under point loads should be exactly the same as for the corresponding "half beams" with prescribed boundary 


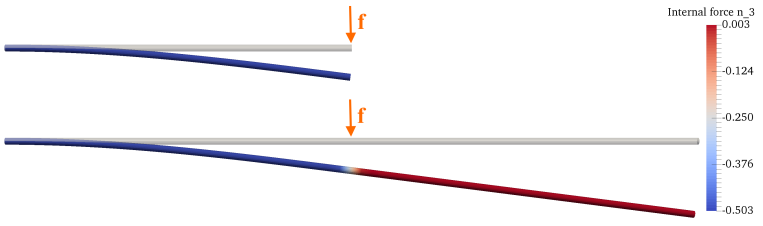

(a) Half and full beams, colored by internal force $n_{3}$

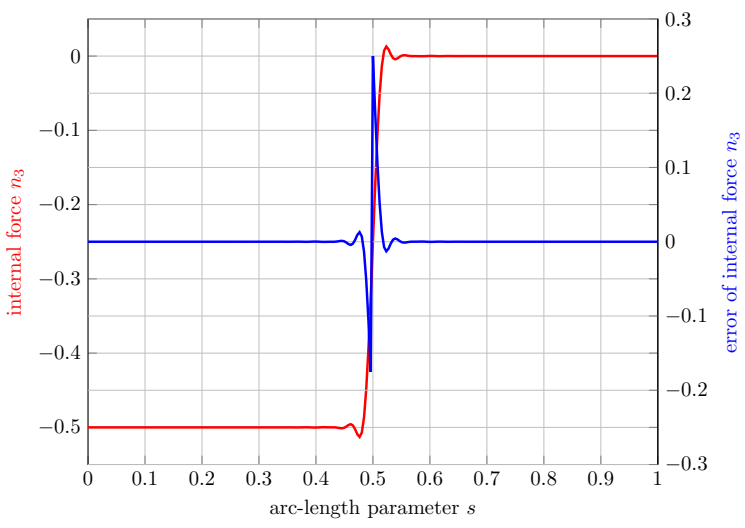

(b) Distribution of internal force $n_{3}$

Figure 6: Cantilever beam evaluation example for the point force method. Results were obtained for $p=8, \ell=64$

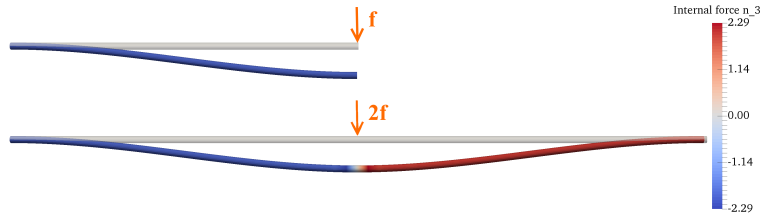

(a) Half and full beams, colored by internal force $n_{3}$

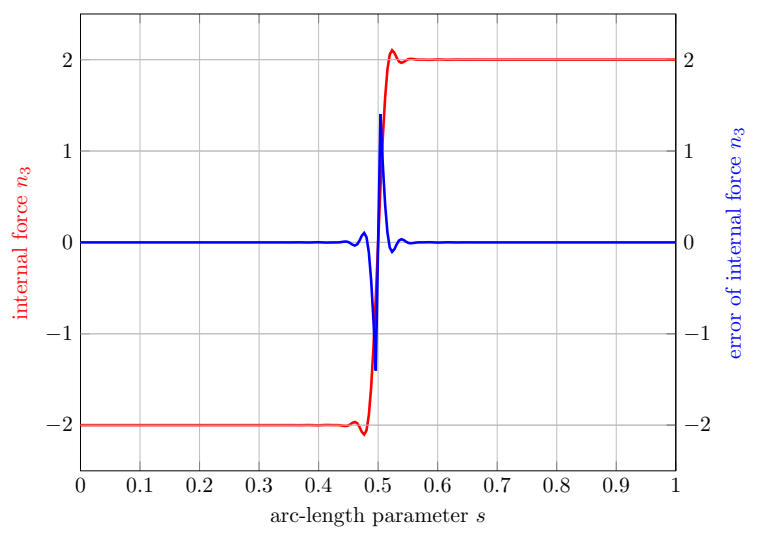

(b) Distribution of internal force $n_{3}$

Figure 7: Double-cantilever beam evaluation example for the point force method. Results were obtained for $p=8, \ell=64$

forces (in the parameter interval $s \in(0,0.5))$. Thus we can use these examples to study the accuracy and convergence of the point load enforcement method.

In Figures $6 \mathrm{a}$ and $7 \mathrm{a}$ the displaced beams are visualized for both cases with a discretization using $p=8, \ell=64$. Visually, there is no difference between the deformed half and full beams, and the internal forces also match. The internal forces $n_{3}$ are plotted over the arc-length parameter $s$ in Figures $6 \mathrm{~b}$ and $7 \mathrm{~b}$ As can be expected, they oscillate around the discontinuity at $s=0.5$, since the formulation requires the stresses to be continuously differentiable, and thus there are relatively high errors compared to the exact solutions, which can inherently not be avoided.

In the convergence plots in Figures 8 and 9 the $L^{2}$-errors of displacements of the "full beams" compared to high accuracy solutions using the "half beams" are shown for the 2-point and 4-point method. For each degree $p$ we have evaluated the error on the interval $s \in(0,0.5)$ for $\ell=2^{k}$ and $\ell=2^{k}+1(k=3, \ldots, 8)$ elements. Since we are applying the force at the center of the "full beams" at $s=0.5$, the point of attack is either right at a collocation point for an odd number of degrees of freedom and collocation points $n=p+\ell$, or in the middle of two collocation points for even $n$. Thus we have separated the convergence plots into evaluation at even number of elements (Figures $8 \mathrm{a}$ and $9 \mathrm{a}$ ) and odd number of elements (Figures $8 \mathrm{~b}$ and 9b.

For the cantilever beam in Fig. 8 , the relative location of the point force with respect to the nearest collocation points $\left(\tau_{k}<\hat{\eta} \leq \tau_{k+1}\right)$ does not have much influence. Initially, we observe higher order convergence rates, but for higher number of elements the order becomes 2 - for both the 2- and 4-point methods.

However, for the double cantilever in Fig. 9 the difference between the force being applied right at and between two collocation points is greater. For the 2-point method we have initially higher order convergence when the point force acts directly at the collocation point, i.e. when the number of collocation points $n=p+\ell$ is odd. This can be observed for $p=5,7$ and even number of elements in Fig. 9a and for $p=4,6,8$ and odd number of elements in Fig. $9 \mathrm{~b}$. For the 4-point method there is no difference between even and odd $n$ and the convergence rate is always 2 . 


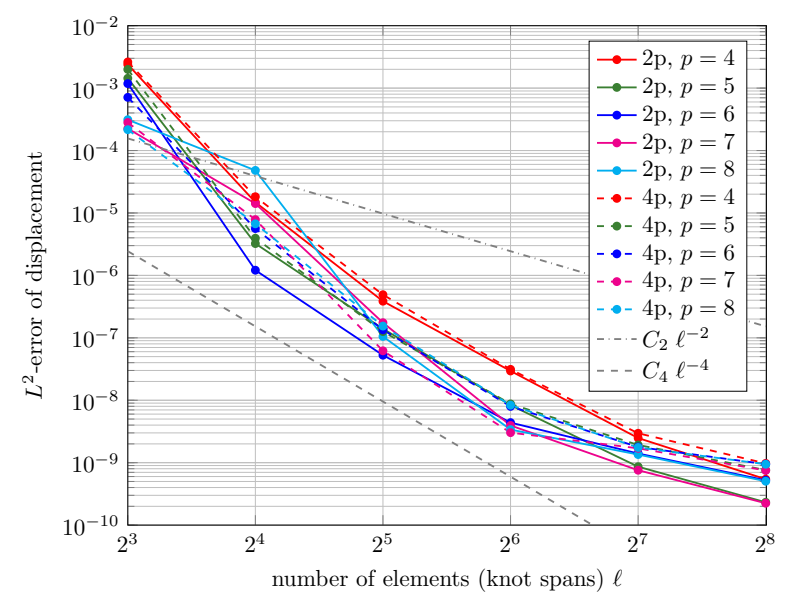

(a) at even number of elements $\ell=2^{k}$

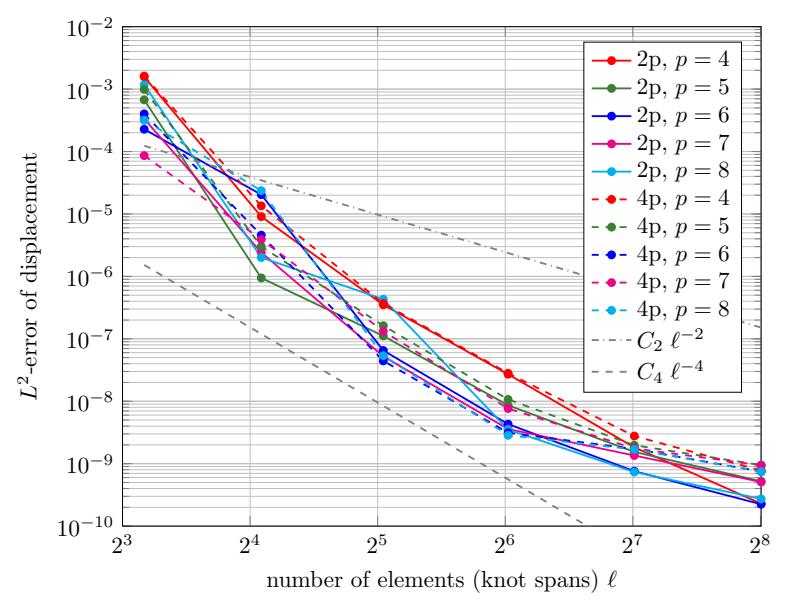

(b) at odd number of elements $\ell=2^{k}+1$

Figure 8: Cantilever beam: convergence of $L^{2}$-error of centerline displacement for the 2-point and 4-point method, evaluated at (a) even and (b) odd number of elements

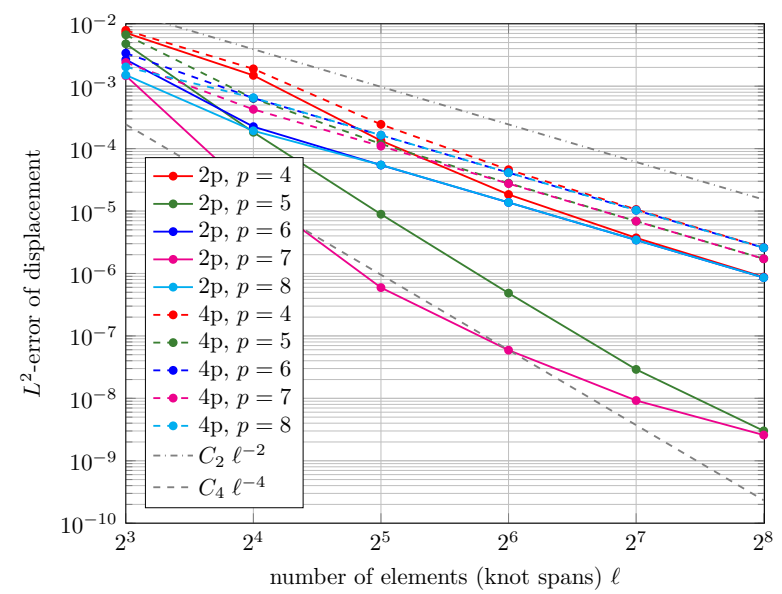

(a) at even number of elements $\ell=2^{k}$

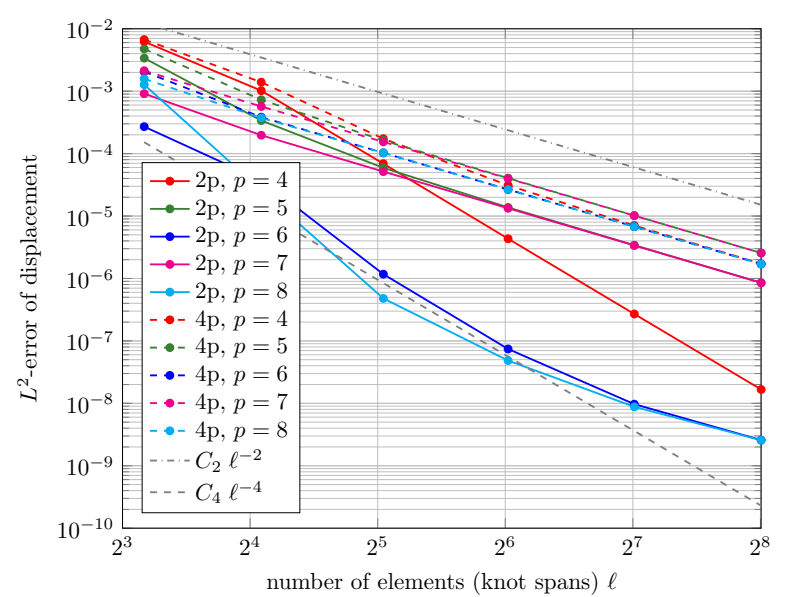

(b) at odd number of elements $\ell=2^{k}+1$

Figure 9: Double-cantilever beam: convergence of $L^{2}$-error of centerline displacement for the 2-point and 4-point method, evaluated at (a) even and (b) odd number of elements

Overall, the convergence studies show that the solutions converge with both methods of introducing the point loads. However, the convergence rates are suboptimal and the accuracy may depend on the scenario and on the relative location of the point of attack with respect to nearest collocation points. In all cases the accuracy of the 2-point method is at least as good as the 4-point method, which makes it more appealing.

\subsection{Evaluation of contact method}

For the evaluation of our contact algorithm and its implementation we study the accuracy and convergence of a benchmark example with two perpendicular, contacting rods, which are shown in their initial and deformed configurations in Fig. 10 .

Both rods are clamped at one end and free at the other, have length $L=0.5$, cross-section radius $r=0.005$, Young's modulus $E=10^{9}$, and Poisson's ratio $\nu=0.5$. They are oriented $90^{\circ}$ with respect to each other and located such that they are initially exactly in contact at parameters $s_{1}=0.733$ and 


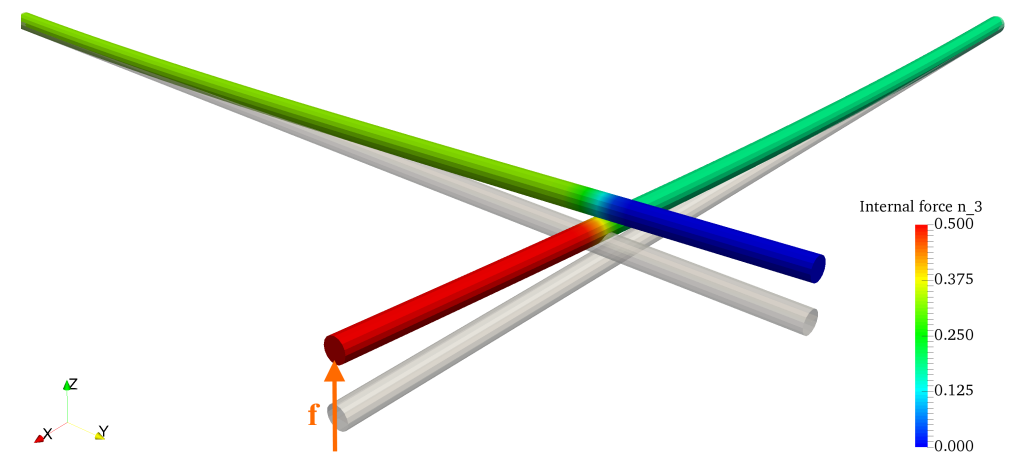

(a) Deformed contacting rods, colored by internal force $n_{3}$. The internal forces are (almost) constant before and after the contact points on both rods

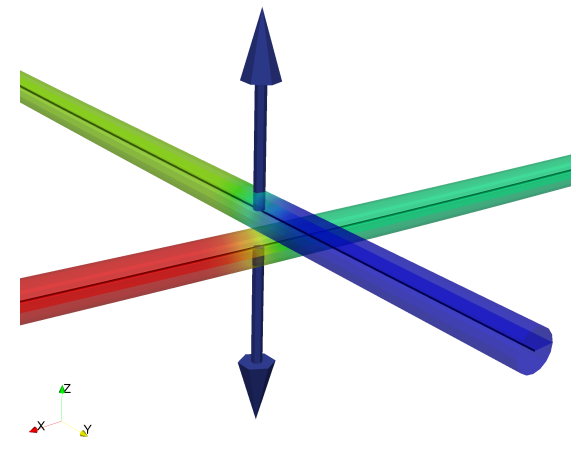

(b) Visualization of the reaction forces $\mathbf{f}_{c}$ at the contact points

Figure 10: Two perpendicular rods example for evaluation of contact method

$s_{2}=0.815$. An upward-directed boundary force $\overline{\mathbf{n}}^{1}(L)=(0,0,0.5)^{\top}$ is prescribed on the free end of the first rod in a single load step, which causes both rods to contact and thus the second rod to deform, too.

We examine the convergence behavior of the rod-to-rod contact with respect to the influence of the following factors: isogeometric discretization, point force method, contact penalty factor, number of contact candidate points, and repetitions of the contact refinement loop. For the contact penalty forces the regularization parameter $p_{\text {reg }}=0.001 r$ is used. We take the $z$-direction displacement, i.e. the displacement out-of-plane of the original position of the rods, at the end of the first rod (where the load is prescribed) as a benchmark. The results are summarized in Fig. 11 .

The influence of the isogeometric discretization, i.e. $p / h / k$-refinement, on the accuracy is investigated in Fig. 11a. From our previous point force study we can conclude that this error is mainly dominated by the accuracy of the enforcement of the point load, rather than the usual discretization error. Here we see that $u_{z}$ converges with increasing number of elements. For low number of elements $\left(\ell \leq 2^{4}\right)$ the overall error is dominated by the discretization error, while for finer meshes $\left(\ell>2^{4}\right)$ it is dominated by the error introduced through the penalty parameter $k_{c}$.

In Sect. 4.3 we have discussed different methods for enforcing the contact forces as point loads: interpolating at 2 collocation points and determining $\lambda$ by integration ( 2 pts., int.), interpolating at 4 collocation points (4 pts., int.), and interpolating at 2 collocation points while approximating $1 / \lambda=J(\hat{\eta})\left(\tau_{k+1}-\tau_{k}\right)$ (2. pts, $J \Delta \tau)$. In Fig. $11 \mathrm{~b}$ we have studied the convergence for $p$ - and $h$-refinement using these 3 methods. All methods converge to the same solution and no method can be identified as clearly superior or inferior to the others. This suggests that we can use the method with 2 collocation points and approximating $\lambda$ (2. pts, $J \Delta \tau)$ for contact forces, as it is computationally the most efficient method, since it does not require performing an integration to determine $\lambda$.

In Fig. 11c we study the influence of the penalty factor $k_{c}$ and the number of contact candidate points $n_{c}$. Note that $L / r=100$, i.e. for 101 equidistant candidate points the step size is equal to the cross-section radius $r$. A higher $n_{c}$ seems to improve the accuracy not significantly, which is reasonable, since we have a refined search for the closest points in our method (provided that $\varepsilon$ in $(16)$ is chosen sufficently large not to miss the relevant contact pair). This finding is important, as the initialization of a contact pair with the closest point search and the computation of the factors for point loads is relatively expensive in our method. Figure $11 \mathrm{c}$ shows that the influence of the penalty factor $k_{c}$ is much more important, and a larger $k_{c}$ improves accuracy. Here we see again that for $k_{c}=10^{4}$ the overall error is dominated by the discretization (point force) error and $n_{c}$ has no significant influence anymore.

All results shown so far were computed with both the contact refinement and Newton loops fully converged. In Fig. 11d we plot the convergence of $u_{z}$ with respect to the number of contact refinement iterations (full loops). Here, a fully converged solution is typically reached after 3-4 iterations. Additionally, the two 


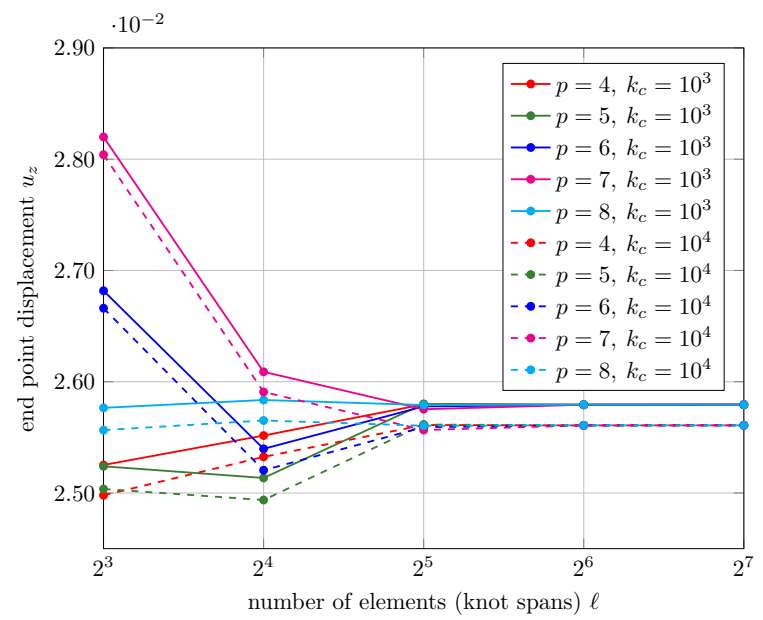

(a) ... for $p / h / k$-refinement with penalty constants $k_{c}=10^{3}$ and $k_{c}=10^{4}\left(n_{c}=21\right)$

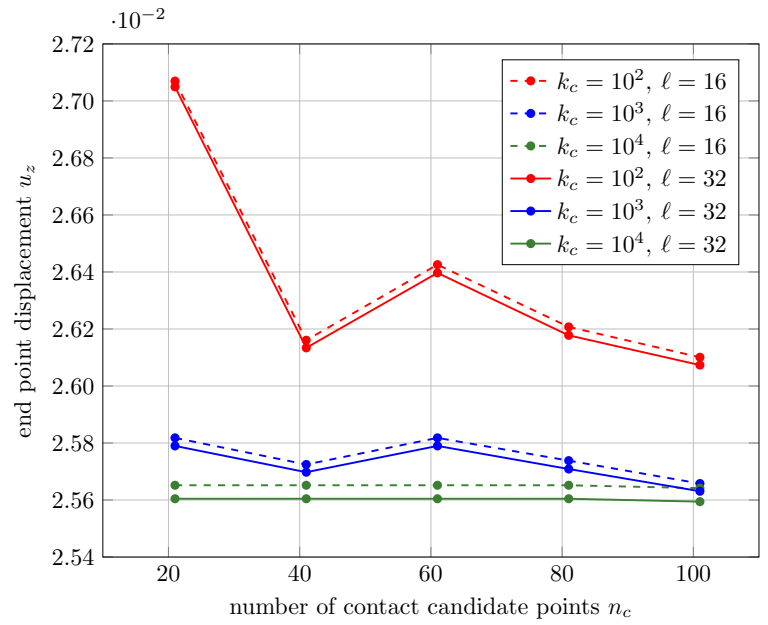

(c) ... for number of equidistant contact candidate points $n_{c}$, comparing values of penalty constant $k_{c}(p=8, \ell=16,32)$

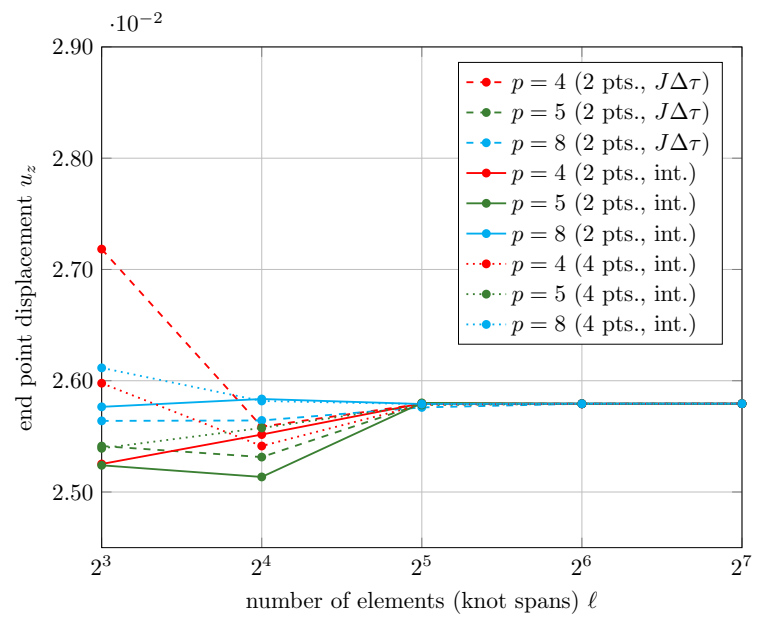

(b) $\ldots$ for point force method $\left(k_{c}=10^{3}, n_{c}=21\right)$

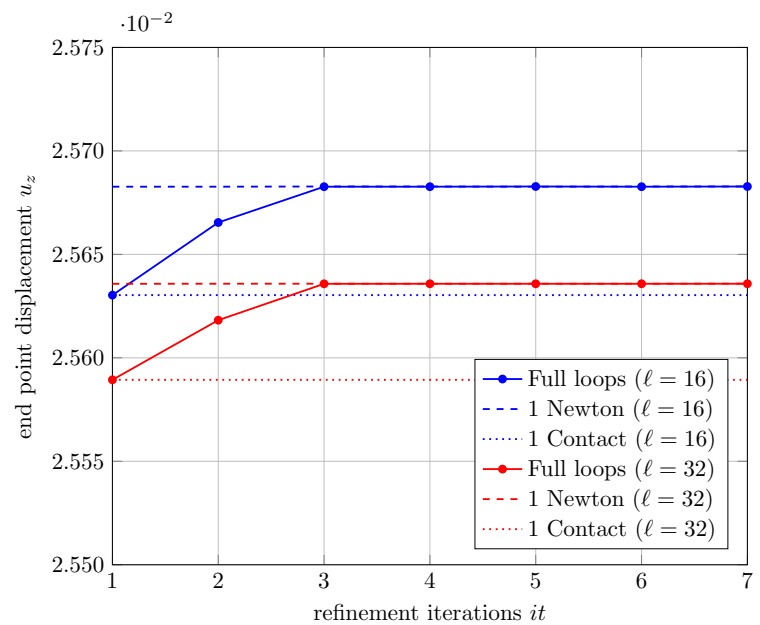

(d) ... for number of contact refinement iterations it ( $p=$ $8, n_{c}=21, k_{c}=10^{-3}$ )

Figure 11: Convergence of $z$-displacement of end point of first rod of two perpendicular, contacting rods ...

edge cases are explored: carrying out only 1 contact refinement step (1 Contact), or only 1 Newton step in each refinement loop (1 Newton). For both cases, the dashed resp. dotted constant lines represent only the final result, not the evolution during each iteration. The latter approach using only 1 Newton step also works very well here, however, it requires a smaller load step to avoid over-shooting and loosing contact.

Overall, this example serves as a validation of our contact implementation and provides a guideline for the choice of different influence factors to efficiently control the accuracy of the contact computation. In order to balance the different errors and computational effort, we suggest using a penalty factor $10^{3} \leq k_{c} \leq 10^{4}$, though the ideal penalty parameter typically depends on application-specific properties such as the material properties and also the mesh, with the simplified 2-point contact force method (2 pts., $J \Delta \tau)$, and limit the contact refinement loop to 3-5 iterations.

\section{Large sliding contact}

To further validate the contact method, we investigate the behavior in large sliding contact. Therefore, we slightly modify the example of two perpendicular rods and shift them such that they are initially exactly 


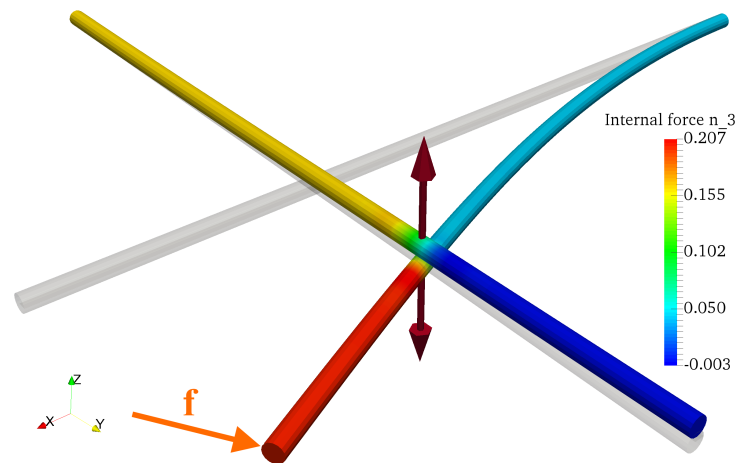

(a) Deformed contacting rods, colored by internal force $\mathbf{n}_{3}$

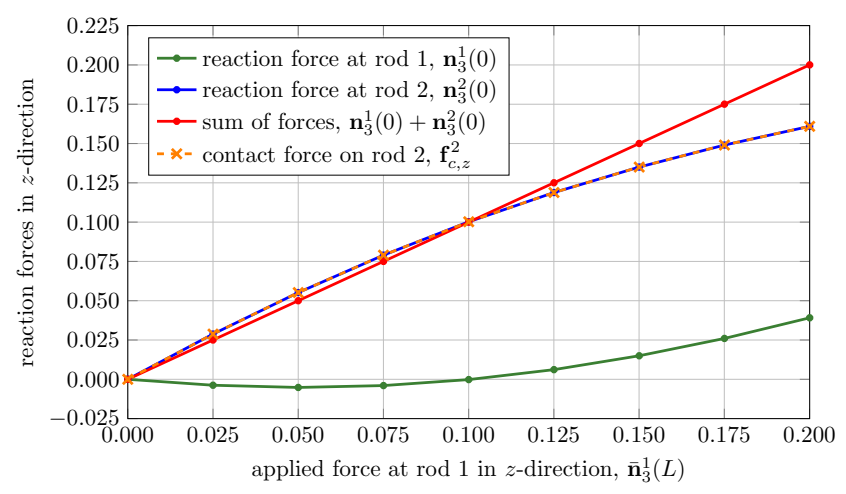

(b) Evaluation of $z$-direction reaction forces over 8 load steps

Figure 12: Two perpendicular rods in large sliding contact

in contact at parameters $s_{1}=0.7$ and $s_{2}=0.5$. Now, a force $\overline{\mathbf{n}}^{1}(L)=(0,2.0,0.2)^{\top}$ is applied at the free end of the lower rod, i.e. rod 1, which pushes the rod slightly upward against rod 2, but mainly bends it in the $y$-direction (around the $z$-axis). Thus, large sliding contact occurs between the two rods, see Fig. 12a.

We simulate the contact deformation process using isogeometric discretizations with $p=6, \ell=32$, contact parameters $n_{c}=41, k_{c}=10^{4}, p_{r e g}=10^{-3} r$ and 2-pts., $J \Delta \tau$-method, and using contact refinement iterations such that the errors and updates are smaller than $10^{-5}$. The force is applied over 8 load steps, which take 3-6 refinement iterations each.

For the evaluation of the method, we have plotted the relevant reaction forces in $z$-direction over the applied external force in $z$-direction, $\overline{\mathbf{n}}_{3}^{1}(L)$, which increases in every load step from 0 to 0.2 , in Figure $12 \mathrm{~b}$. It can be seen that the reaction force at the clamped end on $\operatorname{rod} 2, \mathbf{n}_{3}^{2}(0)$, is equal to the force transmitted onto rod 2 at the contact point, $\mathbf{f}_{c, z}^{2}$, and that the sum of the reaction forces at the clamped ends of both rods, $\mathbf{n}_{3}^{1}(0)+\mathbf{n}_{3}^{2}(0)$, is equal to the force applied at the free end of $\operatorname{rod} 1, \overline{\mathbf{n}}_{3}^{1}(L)$. This shows that the correct contact forces are applied onto the rods by the 2-point force method and that the equilibrium of forces is well maintained (up to an error of $10^{-5}$ ). Thus, the method is able to handle large sliding contacts well.

\subsection{Knot tightening}

In our next, more application-oriented example we simulate a knot tightening process, inspired by [1. We use Young's modulus $E=4 \cdot 10^{9} \mathrm{~Pa}$, Poisson ratio $\nu=0.3$ for the material parameters of the initially straight string with radius $r=0.1 \mathrm{~mm}$, but unlike [1], we divide it in the middle into two rods of length $L_{0}=2 \mathrm{~cm}$, which are both fully clamped at their contact point. This allows us to simulate the process using pure displacement control of the $x$-, $y$ - and $z$ - degrees of freedom of the free ends of both rods, without having to prescribe the end orientations/rotations. For the isogeometric discretization we use $p=8, \ell=64, n=72$ for each rod. The contact parameters are chosen as $n_{c}=61, k_{c}=10^{3} \mathrm{~N} / \mathrm{m}, p_{\text {reg }}=0 \mathrm{~m}$.

The whole knot tightening process is now simulated by prescribing a displacement path for the free ends of the rods, which is divided into 4 phases, see Fig. 13

1. In the bending phase (Fig. 13a) the ends are moved such that the initially straight rods bend (in the $x z$-plane, about the $y$-axis). Small offsets of $\pm r$ are applied in the $y$-direction such that the rods overlap, but do not collide.

2. In the entangling phase (Fig. 13b the ends are moved in the $y$-direction, such that the rods come into contact with each other and their ends entangle. As in 1, two (almost) rigid bars are added to stabilize this process.

3. In the pull-through phase (Fig. 13c) the ends are moved in the $x$-direction until they pass outside the loop. After this phase the two bars are removed.

4. In the final tightening phase (Fig. 13d, 13f) the ends are moved back to the $y=0$ position and at the same time pulled further in the $x$-direction, thus tightening the loop. 


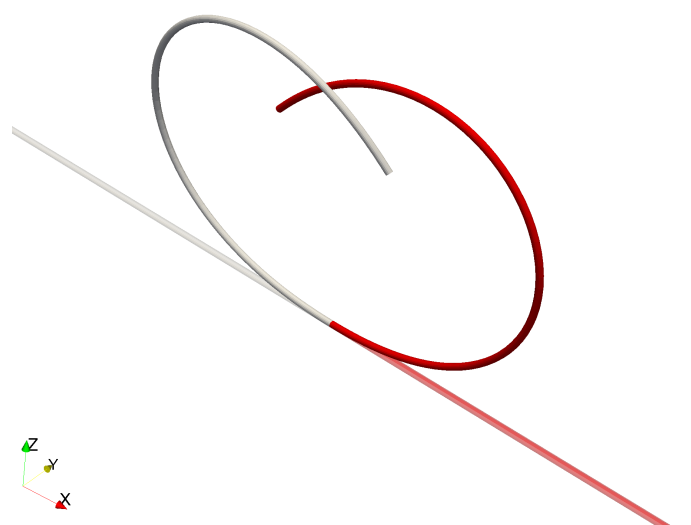

(a) Phase 1 (bending)

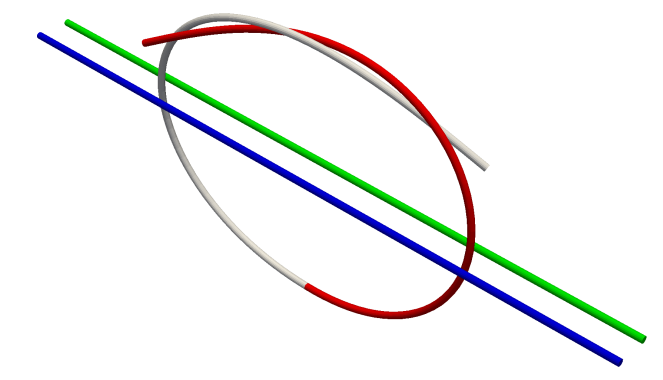

$\frac{x}{2}$

(c) Phase 3 (pull-through)

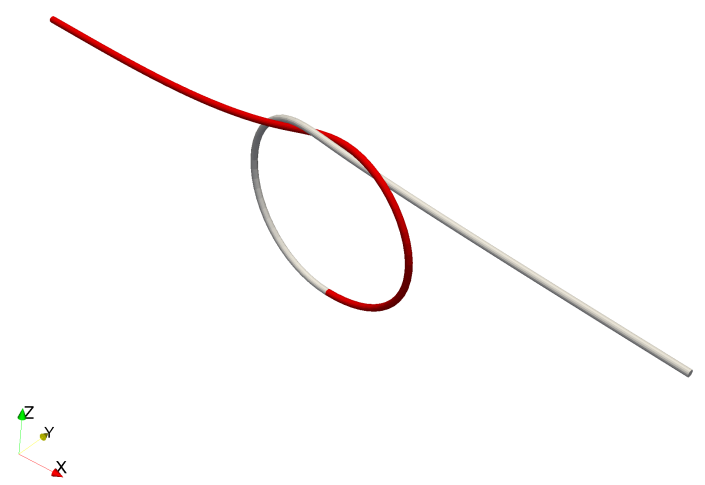

(e) Phase 4(b) (tightening)

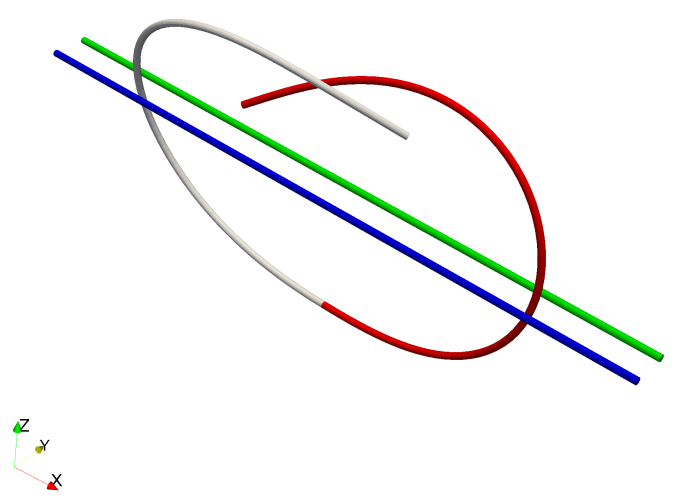

(b) Phase 2 (entangling)
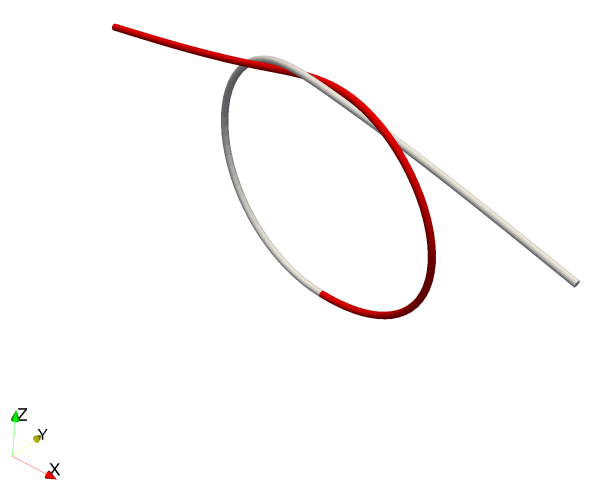

(d) Phase 4(a) (tightening)

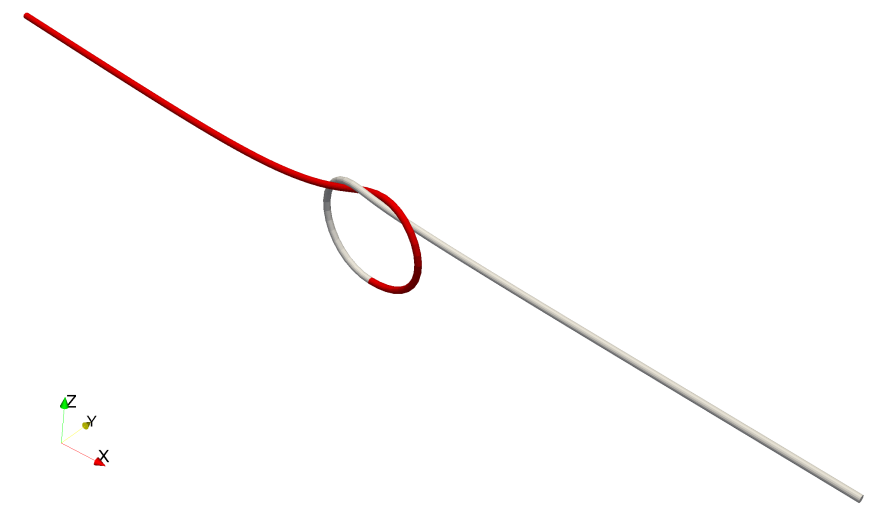

(f) Phase 4(c) (tightening)

Figure 13: Snapshots of knot tightening simulation.

To examine the knot tightening process more closely, Fig. 14 shows close-up views of the rods with contact forces being visualized and Fig. 15 force-displacement curves, where the reaction force at the end point of the left rod and the total sum of absolute values of contact forces are plotted over the $L^{2}$-norm of the displacement of the left rod. In the bending phase, we have a large displacement, but there is no contact yet. Then, in the entangling phase, at first only the two rods, but then also each rod and a bar 


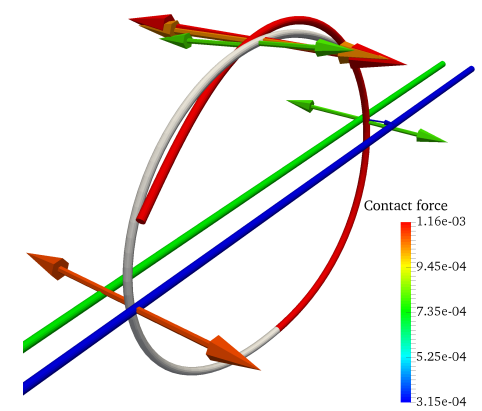

(a) Phase 3, pull-through

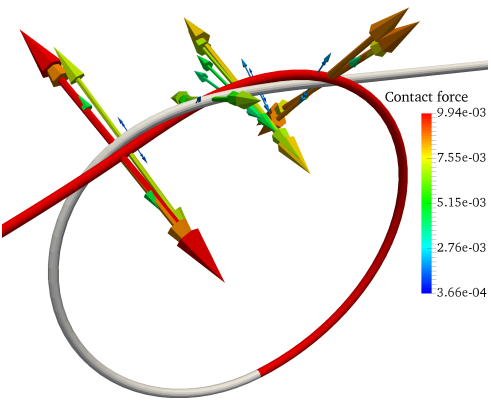

(b) Phase 4(a), tightening

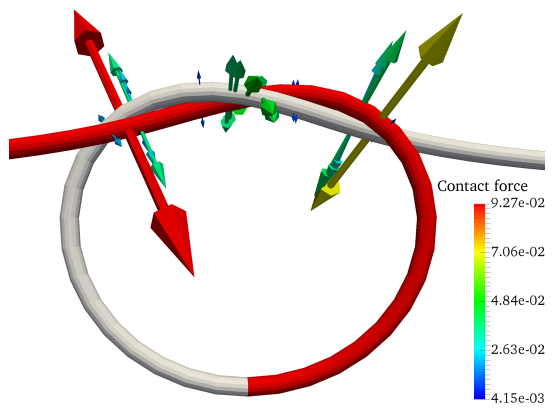

(c) Phase 4(c), tightening

Figure 14: Close-up view of knot tightening with visualization of contact forces (color and length of arrows indicate magnitude of contact force $R_{N}$ )

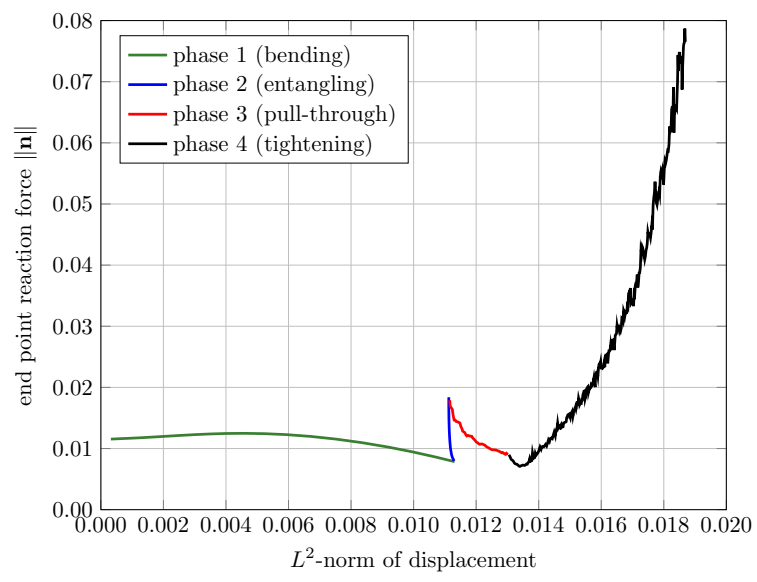

(a) Norm of reaction force $\mathbf{n}$ at end of left rod

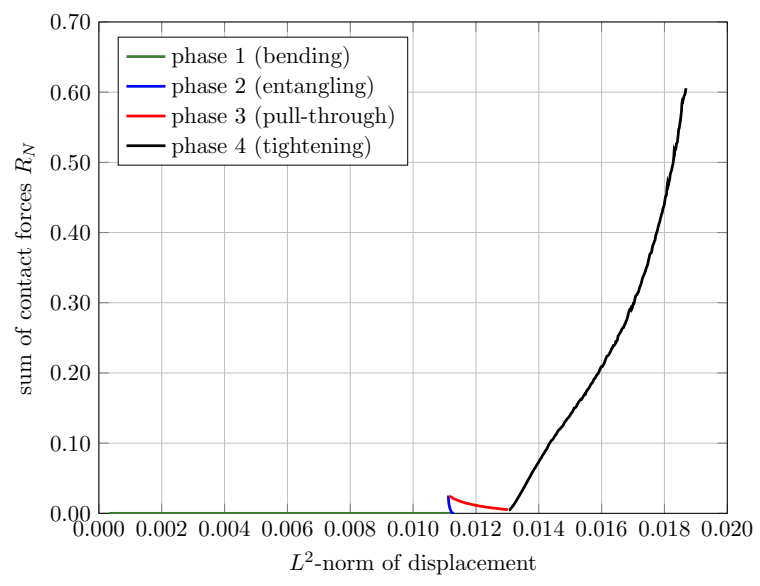

(b) Sum of absolute values of all acting contact forces $R_{N}$

Figure 15: Force-displacement plots for knot tightening simulation. Reaction force at end of left rod (where displacement is prescribed) and sum of absolute values of contact forces are plotted over $L^{2}$-norm of displacement of left rod

come into contact, which leads to a rise in reaction and contact forces. During the pull-through phase, both forces actually drop, which allows us to move away the bars at the end of phase 3. Finally, in the tightening phase, there is a sharp increase of contact and reaction force. There are slight oscillations in the forces, which is due to the sliding contact. Overall, the contact behavior with forces, locations and directions is very reasonable and the results shown in Fig. 14 visually match the ones presented in [1] well.

With this knot tightening application, we were able to solve a highly complex contact problem, where the contact locations and forces change during the deformation process.

\subsection{Woven mesh}

Now we want to move on to a larger scale computational application. We simulate the deformation of a woven mesh under gravity load, see Fig. 16. The mesh consists of a total of 14 rods, which have a cosinusoidal initial shape with 4 periods of oscillation, and are assembled into a textile-like woven $7 \times 7$ mesh. The rods are all clamped at one end and free at the other. The length of one cosinusoidal period is $L_{p}=0.03$, i.e. total length of a rod is $L=4 L_{p}=0.12$, cross-section radius is $r=0.001$, Young's modulus $E=10^{8}$, and Poisson's ratio $\nu=0.5$. The initial mesh is shown in Fig. 16a.

For the simulation, we employ an isogeometric discretization of each rod with degree $p=6$ and $\ell=32$ elements. For the contact discretization we use $n_{c}=41$ equidistantly-spaced candidate points on each rod, 


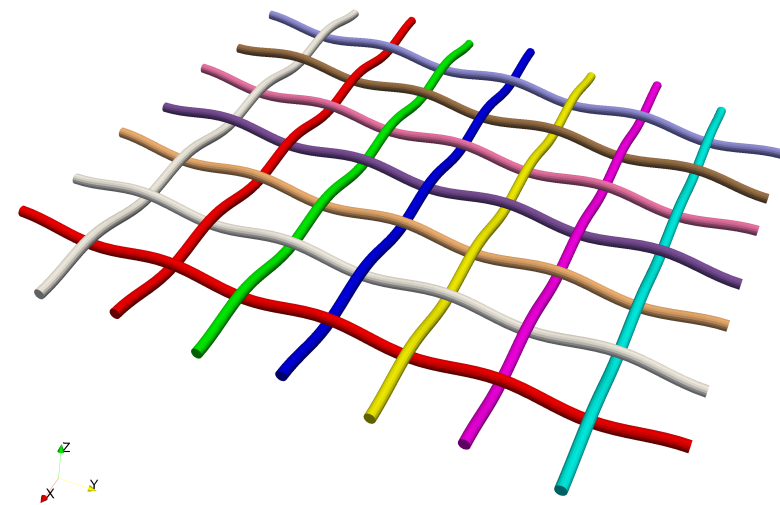

(a) initial structure

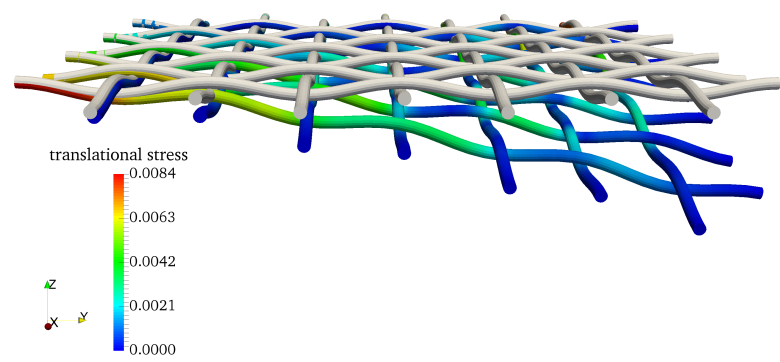

(c) load factor 0.04

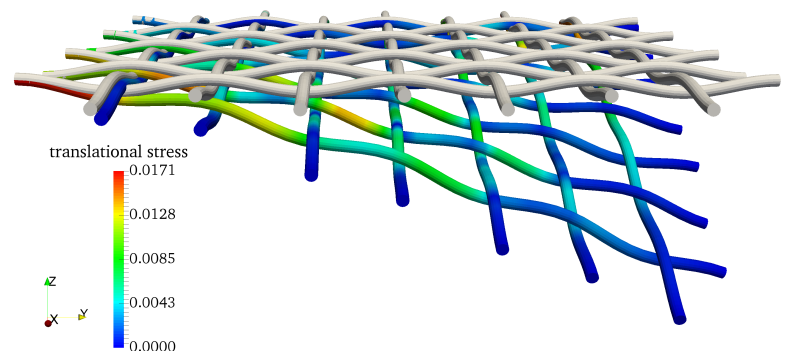

(e) load factor 0.08

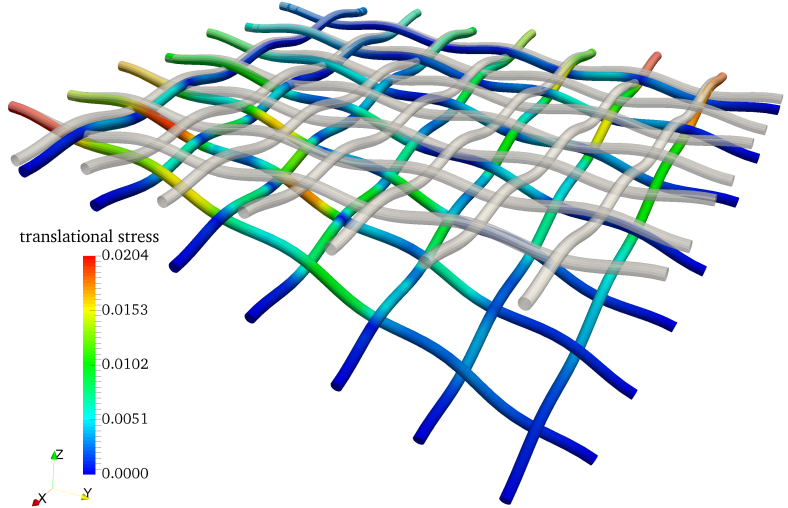

(b) final deformation

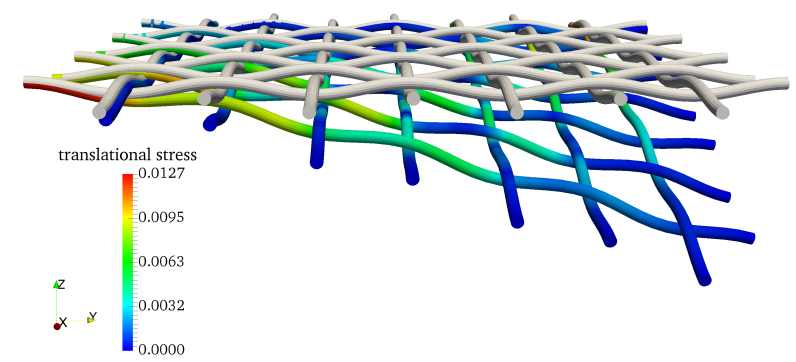

(d) load factor 0.06

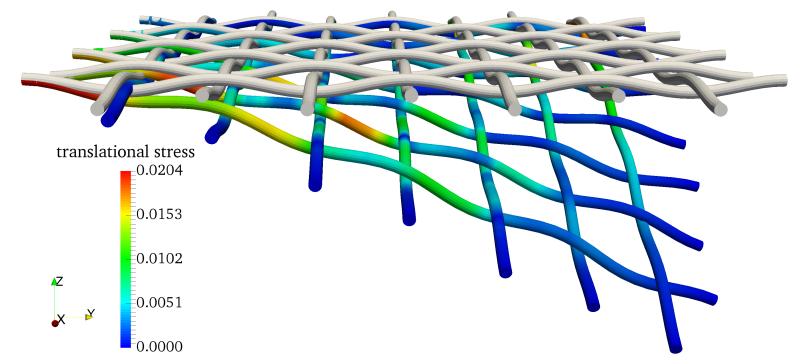

(f) load factor 0.10

Figure 16: Woven mesh deforming under gravity load. Initial and deformed shape are shown in (a) and (b). The deformation process using contact simulation with load increments from 0.002 to $0.1 \mathrm{~N} / \mathrm{m}$ is visualized by four snapshots in (c)-(f)

$k_{c}=10^{3}$ and $p_{\text {reg }}=10^{-6}$. The deformation is induced by a constant, gravity-like line load $\hat{\mathbf{n}}$ on all rods, which acts in the $z$-direction, i.e. perpendicular to the plane of the mesh, and is increased in 49 load steps from 0.002 to $0.1 \mathrm{~N} / \mathrm{m}$.

The deformed mesh at the end of the simulation is shown in Fig. $16 \mathrm{~b}$ and four snapshots of the deformation for load factors $0.04,0.06,0.08$ and 0.10 are given in Fig. 16c 16f Due to the mesh structure, there should be up to 49 possible contact locations, of which usually more than 40 were active after convergence of each load step (i.e. there was a slight penetration at these locations). To keep the penetrations reasonably small, we have gradually increased $k_{c}$ by multiplying it with a factor proportional to the load factor, here from 1 to 50 . In this way the penetrations after the final load step are kept in the order of $10^{-7}$, i.e. $10^{-4}$ times the radius $r$.

Overall, with this application we could show that our contact formulation is able to handle larger scale examples with multiple contact interactions. 


\section{Summary and conclusions}

We proposed a frictionless contact formulation for geometrically nonlinear spatial rods within the framework of isogeometric collocation using NURBS discretizations for the geometry and unknown fields. In the collocation approach, the linear and angular momentum balance equations as well as the Neumann boundary conditions are discretized and collocated in strong form. The frictionless contact formulation encompasses the geometric contact detection phase and the computation of the contact forces, which stems from the enforcement of the contact constraints using the penalty method. The factors affecting the accuracy of the results, and in particular a suitable strategy for introducing point loads within the collocation scheme, are analyzed in detail, along with algorithmic aspects of the proposed formulation. It is shown that the order of spatial convergence of the proposed scheme is affected by the introduction of concentrated contact forces at points not necessarily coinciding with collocation points. Several numerical applications demonstrate that the proposed formulation is a feasible, accurate and robust approach for contact computations within the isogeometric collocation framework. In further work, we are planning to extend the contact formulation to the frictional case, and to address thin rod models and dynamic problems.

\section{Acknowledgements}

O.W., B.N. and M.L.D. acknowledge support from the SUTD Digital Manufacturing and Design Centre, supported by the Singapore National Research Foundation. L.D.L. was partially supported by the European Research Council through the Starting Grant program (GA 279439), and the DFG Priority Program SPP 1748 "Reliable Simulation Techniques in Solid Mechanics". J.K. was partially supported by the Onsager Fellowship Programme of NTNU.

\section{Bibliography}

[1] D. Durville. Contact-friction modeling within elastic beam assemblies: an application to knot tightening. Computational Mechanics, 49(6):687-707, 2012.

[2] D. Durville. Simulation of the mechanical behaviour of woven fabrics at the scale of fibers. International Journal of Material Forming, 3:1241-1251, 2010

[3] D. Durville. Numerical simulation of entangled materials mechanical properties. Journal of Materials Science, 40:5941$5948,2005$.

[4] A. Torre, H. Bajas, D. Ciazynski, D. Durville, and K. Weiss. Mechanical-electrical modeling of stretching experiment on 45 Nb3Sn strands CICCs. IEEE Transactions on Applied Superconductivity, 21:2042-2045, 2011.

[5] O. Weeger, Y.S.B. Kang, S.-K. Yeung, and M.L. Dunn. Optimal design and manufacture of active rod structures with spatially variable materials. 3D Printing and Additive Manufacturing, 3(4):204-215, 2016.

[6] T. J. R. Hughes, J. A. Cottrell, and Y. Bazilevs. Isogeometric analysis: CAD, finite elements, NURBS, exact geometry and mesh refinement. Computer Methods in Applied Mechanics and Engineering, 194(39-41):4135-4195, 2005.

[7] J.A. Cottrell, A. Reali, Y. Bazilevs, and T.J.R. Hughes. Isogeometric analysis of structural vibrations. Computer Methods in Applied Mechanics and Engineering, 195(41-43):5257-5296, 2006.

[8] O. Weeger, U. Wever, and B. Simeon. Isogeometric analysis of nonlinear Euler-Bernoulli beam vibrations. Nonlinear Dynamics, 72(4):813-835, 2013.

[9] L. Greco and M. Cuomo. B-spline interpolation of Kirchhoff-Love space rods. Computer Methods in Applied Mechanics and Engineering, 256:251-269, 2013.

[10] A.M. Bauer, M. Breitenberger, B. Philipp, R. Wüchner, and K.-U. Bletzinger. Nonlinear isogeometric spatial Bernoulli beam. Computer Methods in Applied Mechanics and Engineering, 303:101-127, 2016.

[11] R. Bouclier, T. Elguedj, and A. Combescure. Locking free isogeometric formulations of curved thick beams. Computer Methods in Applied Mechanics and Engineering, 245-246:144-162, 2012.

[12] J. Kiendl, F. Auricchio, T.J.R. Hughes, and A. Reali. Single-variable formulations and isogeometric discretizations for shear deformable beams. Computer Methods in Applied Mechanics and Engineering, 284:988-1004, 2015.

[13] N. Cavallini, O. Weeger, M. S. Pauletti, M. Martinelli, and P. Antolín. Effective integration of sophisticated operators in isogeometric analysis with igatools. In B. Jüttler and B. Simeon, editors, Isogeometric Analysis and Applications 2014, volume 107 of Lecture Notes in Computational Science and Engineering, pages 209-230. Springer International Publishing, 2015.

[14] B. Jüttler, U. Langer, A. Mantzaflaris, S.E. Moore, and W. Zulehner. Geometry + Simulation Modules: Implementing Isogeometric Analysis. PAMM, 14(1):961-962, 2014.

[15] F. Auricchio, L. Beirão da Veiga, T. J. R. Hughes, A. Reali, and G. Sangalli. Isogeometric collocation methods. Mathematical Models and Methods in Applied Sciences, 20(11):2075-2107, 2010. 
[16] A. Reali and T.J.R. Hughes. An introduction to isogeometric collocation methods. In G. Beer and S. Bordas, editors, Isogeometric Methods for Numerical Simulation, volume 561 of CISM International Centre for Mechanical Sciences, pages 173-204. Springer, 2015.

[17] C. Anitescu, Y. Jia, J. Zhang, and T. Rabczuk. An isogeometric collocation method using superconvergent points. Computer Methods in Applied Mechanics and Engineering, 284:1073-1097, 2015.

[18] H. Gomez and L. De Lorenzis. The variational collocation method. Computer Methods in Applied Mechanics and Engineering, 309:152-181, 2016.

[19] F. Auricchio, L. Beirão da Veiga, T.J.R. Hughes, A. Reali, and G. Sangalli. Isogeometric collocation for elastostatics and explicit dynamics. Computer Methods in Applied Mechanics and Engineering, 249-252:2-14, 2012.

[20] D. Schillinger, J.A. Evans, A. Reali, M.A. Scott, and T.J.R. Hughes. Isogeometric collocation: Cost comparison with Galerkin methods and extension to adaptive hierarchical NURBS discretizations. Computer Methods in Applied Mechanics and Engineering, 267:170-232, 2013.

[21] L. Beirão da Veiga, C. Lovadina, and A. Reali. Avoiding shear locking for the Timoshenko beam problem via isogeometric collocation methods. Computer Methods in Applied Mechanics and Engineering, 241-244:38-51, 2012.

[22] F. Auricchio, L. Beirão da Veiga, J. Kiendl, C. Lovadina, and A. Reali. Locking-free isogeometric collocation methods for spatial Timoshenko rods. Computer Methods in Applied Mechanics and Engineering, 263:113-126, 2013.

[23] J. Kiendl, F. Auricchio, L. Beirão da Veiga, C. Lovadina, and A. Reali. Isogeometric collocation methods for the ReissnerMindlin plate problem. Computer Methods in Applied Mechanics and Engineering, 284:489-507, 2015.

[24] A. Reali and H. Gomez. An isogeometric collocation approach for Bernoulli-Euler beams and Kirchhoff plates. Computer Methods in Applied Mechanics and Engineering, 284:623-636, 2015. Isogeometric Analysis Special Issue.

[25] L. De Lorenzis, J.A. Evans, T.J.R. Hughes, and A. Reali. Isogeometric collocation: Neumann boundary conditions and contact. Computer Methods in Applied Mechanics and Engineering, 284:21-54, 2015.

[26] R. Kruse, N. Nguyen-Thanh, L. De Lorenzis, and T.J.R. Hughes. Isogeometric collocation for large deformation elasticity and frictional contact problems. Computer Methods in Applied Mechanics and Engineering, 296:73-112, 2015.

[27] O. Weeger, S.-K. Yeung, and M.L. Dunn. Isogeometric collocation methods for Cosserat rods and rod structures. Computer Methods in Applied Mechanics and Engineering, 316(Special Issue on Isogeometric Analysis):100-122, 2017.

[28] E. Marino. Isogeometric collocation for three-dimensional geometrically exact shear-deformable beams. Computer Methods in Applied Mechanics and Engineering, 307:383-410, 2016.

[29] L. De Lorenzis, P. Wriggers, and T.J.R. Hughes. Isogeometric contact: a review. GAMM-Mitteilungen, 37(1):85-123, 2014.

[30] P. Wriggers and G. Zavarise. On contact between three-dimensional beams undergoing large deflections. Communications in Numerical Methods in Engineering, 13:429-1438, 1997.

[31] G. Zavarise and P. Wriggers. Contact with friction between beams in 3-D space. International Journal for Numerical Methods in Engineering, 49(8):977-1006, 2000.

[32] P. Litewka and P. Wriggers. Contact between 3D beams with rectangular cross-sections. International Journal for Numerical Methods in Engineering, 53:2019-2041, 2002.

[33] P. Litewka and P. Wriggers. Frictional contact between 3d beams. Computational Mechanics, 28:26-39, 2002.

[34] A. Konyukhov and K. Schweizerhof. Computational Contact Mechanics: Geometrically Exact Theory for Arbitrary Shaped Bodies, volume 67 of Lecture Notes in Applied and Computational Mechanics. Springer-Verlag Berlin Heidelberg, 2013.

[35] A.G. Neto, P.M. Pimenta, and P. Wriggers. A master-surface to master-surface formulation for beam to beam contact. Part I: frictionless interaction. Computer Methods in Applied Mechanics and Engineering, 303:400-429, 2016.

[36] M. Chamekh, S. Mani-Aouadi, and M. Moakher. Modeling and numerical treatment of elastic rods with frictionless self-contact. Computer Methods in Applied Mechanics and Engineering, 198(47-48):3751-3764, 2009.

[37] P. Litewka. Multiple-point beam-to-beam contact finite element. In Proceedings of CMM-2011, Warsaw, Poland, 2011.

[38] C. Meier, A. Popp, and W. A. Wall. A finite element approach for the line-to-line contact interaction of thin beams with arbitrary orientation. Computer Methods in Applied Mechanics and Engineering, 308:377-413, 2016.

[39] C. Meier, W. A. Wall, and A. Popp. A unified approach for beam-to-beam contact. Computer Methods in Applied Mechanics and Engineering, 315:972-1010, 2017.

[40] M. Chamekh, S. Mani-Aouadi, and M. Moakher. Stability of elastic rods with self-contact. Computer Methods in Applied Mechanics and Engineering, 279:227-246, 2014.

[41] A.G. Neto, P.M. Pimenta, and P. Wriggers. Self-contact modeling on beams experiencing loop formation. Computational Mechanics, 55:193-208, 2015.

[42] S. Eugster. Geometric Continuum Mechanics and Induced Beam Theories, volume 75 of Lecture Notes in Applied and Computational Mechanics. Springer International Publishing, 2015.

[43] S.S. Antman. Nonlinear Problems of Elasticity, volume 107 of Applied Mathematical Sciences. Springer New York, 2005.

[44] J.C. Simo. A finite strain beam formulation. The three-dimensional dynamic problem. Part I. Computer Methods in Applied Mechanics and Engineering, 49(1):55-70, 1985.

[45] J. A. Cottrell, T. J. R. Hughes, and Y. Bazilevs. Isogeometric Analysis: Toward Integration of CAD and FEA. John Wiley \& Sons, Ltd, 2009.

[46] L. A. Piegl and W. Tiller. The NURBS Book. Monographs in Visual Communication. Springer, 1997.

[47] P. Wriggers. Computational Contact Mechanics. Springer-Verlag Berlin Heidelberg, 2006.

[48] C. Ericson. Real-time collision detection. CRC Press, 2004.

[49] H. Casquero, L. Liu, Y. Zhang, A. Reali, and H. Gomez. Isogeometric collocation using analysis-suitable T-splines of arbitrary degree. Computer Methods in Applied Mechanics and Engineering, 301:164-186, 2016. 